\title{
On the Algebra of Field Operators. The Weak Commutant and Integral Decompositions of States
}

\author{
H. J. Borchers and J. Yngvason \\ Institut für Theoretische Physik, Universität Göttingen, Göttingen, Federal Republic of Germany
}

Received July 15, 1974; in revised form January 29, 1975

\begin{abstract}
It is shown that every continuous representation of a nuclear and separable *-algebra on a separable Hilbert space has a certain integral decomposition into representations with a trivial weak commutant. This result is used to obtain a decomposition of Wightman functionals into extremal states.

An example is given of an extremal Wightman functional which does not have the cluster property.
\end{abstract}

\section{Introduction}

This paper deals with integral decompositions of representations and states on the algebra of test functions for quantum fields [1-3]. In comparison with the reduction theory for $C^{*}$ - and v. Neumann algebras one has to face some new problems. To begin with, the positive cone in the dual space of the algebra in question does not have a compact base, so the usual integral representation theory on compact convex sets [4] does not apply. Although it is possible to use the theory of conical measures $[4,5]$ it is not at all clear whether a maximal conical measure is concentrated on the extremal rays in the dual cone in some sense. Therefore, it seems more promising for a decomposition of a state into extremal states to look at the corresponding representation and decompose with respect to an abelian algebra in the commutant. This is in principle the method we shall use, but there is, however, an essential complication. The operators we have to deal with are in general unbounded, so one has to distinguish between two notions of commutativity, strong and weak. For the decomposition into extremal states, the weak commutant is relevant, because a state is extremal if and only if it defines a representation with a trivial weak commutant. However, unlike the strong commutant, the weak commutant is in general not an algebra, and a decomposition with respect to a maximal abelian algebra in the weak commutant will mostly not result in a decomposition into extremal states. A simple example where this problem occurs is the case of one Hermitean operator with a nonsymmetrical defect index. The solution is well known (see e.g. [6], Appendix I). One considers a self adjoint extension of the operator in a larger Hilbert space and decomposes this extended operator. Our program is to do a similar thing for families of unbounded operators.

Whereas the existence of a suitable extension for one operator is a rather simple matter [6], the main problem we have to solve is to construct such an 
extension for a whole family of operators. This will be done in the next section. Once we have obtained this result (Theorem 2.16), we apply methods analogous to those of Maurin [7] to get an integral decomposition. Apart from separability assumptions, essentially the only new ingredient needed is a nuclear topology on the domain of the operators. We show in particular, that every state on a nuclear, separable and barrelled *-algebra can be decomposed into extremal states. In the last section we discuss the implications for Wightman field theory. We consider also the question whether extremal Wightman states do always have the cluster property and find the answer to be negative.

The problem of obtaining an integral decomposition of Wightman states has been treated by Maurin [7, 8] and for the $P(\Phi)_{2}$-model by Bratteli [9]. In [7, 8] the states are decomposed with respect to a maximal abelian algebra in the weak commutant. The spectral theorem for nuclear spaces ensures that the states appearing in the decomposition consist of tempered distributions if this is the case for the original state. As already mentioned, such a decomposition is not necessarily a decomposition into extremal states, but in the cases where strong and weak commutant coincide, this will be so. In particular one could apply this theory to the $P(\Phi)_{2}$-model. The results in [9] are obtained with different methods and give more detailed information for this particular case.

\section{Extension Theory}

This section is concerned with families of unbounded operators on Hilbert spaces and their commutants. The main result is Theorem 2.16, which provides the basis for the decomposition theory in the next section.

2.1. Definition. A $*$-operator family is defined as a pair $(\mathscr{A}, \mathscr{D})$ with the following properties.

(i) $\mathscr{D}$ is a pre-Hilbert space with completion $\mathscr{H}(\mathscr{D})$.

(ii) $\mathscr{A}$ is a family of linear operators defined on $\mathscr{D}$ with values in $\mathscr{H}(\mathscr{D})$, subject to the condition that for every $A \in \mathscr{A}$ there is an $A^{*} \in \mathscr{A}$ such that $\langle\varphi, A \psi\rangle$ $=\left\langle A^{*} \varphi, \psi\right\rangle$ for all $\varphi, \psi \in \mathscr{D}$.

2.2. Remarks. (1) From the last assumption it follows that every $A \in \mathscr{A}$ is a closable operator on $\mathscr{H}(\mathscr{D})$.

(2) It is not assumed at this point that $\mathscr{D}$ is complete in any topology, in particular not in the topology induced by the graph norms of the operators $A \in \mathscr{A}$.

The concept of an extension, well known for one operator, generalizes to a family of operators as follows:

2.3. Definition. A $*$-operator family $(\hat{\mathscr{A}}, \hat{\mathscr{D}})$ will be called an extension of $(\mathscr{A}, \mathscr{D})$ if

(i) $\hat{\mathscr{D}} \supset \mathscr{D}$ and the norm defined on $\hat{\mathscr{D}}$ coincides on $\mathscr{D}$ with the norm on $\mathscr{D}$.

(ii) There is a bijective mapping $A \mapsto \hat{A}$ from $\mathscr{A}$ onto $\hat{\mathscr{A}}$, preserving all algebraic operations and the involution $*$, such that the restriction $\hat{A} \uparrow \mathscr{D}$ is equal to $A$ for all $A \in \mathscr{A}$.

2.4. Remarks. (1) By this definition of an extension the Hilbert spaces $\mathscr{H}(\mathscr{D})$ and $\mathscr{H}(\hat{\mathscr{D}})$ do not necessarily coincide, but since $\mathscr{D} \subset \hat{\mathscr{D}}$, the Hilbert space $\mathscr{H}(\mathscr{D})$ is naturally imbedded as a closed subspace of $\mathscr{H}(\hat{\mathscr{D}})$. 
(2) We can always obtain an extension by completing $\mathscr{D}$ in the topology induced by the graph norms of the operators in $\mathscr{A}$. (If $\mathscr{D}$ is not already complete in this topology.) If we denote this extension by $(\overline{\mathscr{A}}, \overline{\mathscr{D}})$, then clearly $\mathscr{H}(\mathscr{D})=\mathscr{H}(\overline{\mathscr{D}})$. This estension, however, is only of limited interest except as a technical device for proofs. We shall refer to it as the closure of $(\mathscr{A}, \mathscr{D})$.

Since we have in general to deal with unbounded operators we must distinguish between the strong and the weak commutant of an operator family.

2.5. Definition. (1) $(\mathscr{A}, \mathscr{D})_{w}^{\prime}$ denotes the set of all bounded linear operators $b$ acting on $\mathscr{H}(\mathscr{D})$ with the property

$$
\langle b \varphi, A \psi\rangle=\left\langle A^{*} \varphi, b^{*} \psi\right\rangle
$$

for all $\varphi, \psi \in \mathscr{D}, A \in \mathscr{A}$.

(2) $(\mathscr{A}, \mathscr{D})_{s}^{\prime}$ denotes the set of all bounded linear operators $b$ on $\mathscr{H}(\mathscr{D})$ such that

(i) $b \mathscr{D} \subset \mathscr{D}$.

(ii) For every $A \in \mathscr{A}$ and $\varphi \in \mathscr{D}$ we have $b A \varphi=A b \varphi$.

(3) $(\mathscr{A}, \mathscr{D})_{s}^{\prime}$ resp. $(\mathscr{A}, \mathscr{D})_{w}^{\prime}$ is called the strong resp. weak commutant of $(\mathscr{A}, \mathscr{D})$. The properties of these sets are listed in the following.

2.6. Lemma. (i) $(\mathscr{A}, \mathscr{D})_{w}^{\prime}$ is a weakly closed linear subspace of the bounded operators on $\mathscr{H}(\mathscr{D})$ containing the unit operator 1.

(ii) $(\mathscr{A}, \mathscr{D})_{w}^{\prime}$ is $*$-invariant.

(iii) $(\mathscr{A}, \mathscr{D})_{w}^{\prime}$ is the linear span of its positive elements.

(iv) $(\mathscr{A}, \mathscr{D})_{w}^{\prime}=(\overline{\mathscr{A}}, \overline{\mathscr{D}})_{w}^{\prime}$.

(v) $(\mathscr{A}, \mathscr{D})_{s}^{\prime}$ is an algebra.

(vi) $(\overline{\mathscr{A}}, \overline{\mathscr{D}})_{s}^{\prime}$ is weakly closed.

(vii) $(\mathscr{A}, \mathscr{D})_{s}^{\prime} \subset(\mathscr{A}, \mathscr{D})_{w}^{\prime}$.

Proof. The statements (i), (iv), (v), and (vii) are more or less trivial. (ii): $\mathscr{A}$ is invariant under the operation of taking adjoints and restricting to $\mathscr{D}$. Therefore, if $b \in(\mathscr{A}, \mathscr{D})_{w}^{\prime}, A \in \mathscr{A}$ and $\varphi, \psi \in \mathscr{D}$, we have

$$
\left\langle b^{*} \varphi, A \psi\right\rangle=\left\langle b^{*} \varphi,\left(A^{*}\right)^{*} \psi\right\rangle=\left\langle A^{*} \varphi, b \psi\right\rangle=\left\langle A^{*} \varphi, b^{* *} \psi\right\rangle,
$$

so $b^{*} \in(\mathscr{A}, \mathscr{D})_{w}^{\prime}$.

(iii) From (ii) it follows that $(\mathscr{A}, \mathscr{D})_{w}^{\prime}$ is generated by its Hermitean elements. Moreover, if $b=b^{*} \in(\mathscr{A}, \mathscr{D})_{w}^{\prime}$, we have $\|b\| \cdot 1 \pm b \in(\mathscr{A}, \mathscr{D})_{w}^{\prime}, \quad\|b\| \cdot 1 \pm b \geqq 0$ and $b=\frac{1}{2}(\|b\| \cdot 1+b)-\frac{1}{2}(\|b\| \cdot 1-b)$, so $(\mathscr{A}, \mathscr{D})_{w}^{\prime}$ is also the linear span of its positive elements.

(vi): Suppose $\left\{b_{\alpha}\right\}$ is a net in $(\mathscr{A}, \mathscr{D})_{s}^{\prime}$ converging weakly to a bounded operator $b$. If $A \in \mathscr{A}$ and $\varphi \in \mathscr{D}$ we have $b_{\alpha} A \varphi=A b_{\alpha} \varphi$, so $\left(b_{\alpha} \varphi, b_{\alpha} A \varphi\right)$ belongs to the graph of $A$. If $b_{\alpha} \rightarrow b$, this converges weakly to $(b \varphi, b A \varphi)$. For linear subsets strong and weak closure are identical, so $(b \varphi, b A \varphi)$ is in the graph of the closure of $A$ which proves the statement.

2.7. Remarks. (1) $(\mathscr{A}, \mathscr{D})_{w}^{\prime}$ is in general not an algebra, cf. [10], Lemma 3.2. (2) $(\mathscr{A}, \mathscr{D})_{s}^{\prime}$ is in general not a $*$-algebra, even not when $(\mathscr{A}, \mathscr{D})_{s}^{\prime}$ is weakly closed. (3) If the family $(\mathscr{A}, \mathscr{D})$ is self-adjoint in the sense of [10] (i.e. if $\mathscr{D}$ equals the intersection of the domains of all adjoints of the operators in $\mathscr{A}$ ) then we have $(\mathscr{A}, \mathscr{D})_{w}^{\prime}=(\mathscr{A}, \mathscr{D})_{s}^{\prime}$. (Cf. [10], Lemmas 4.5 and 4.6.) In general the strong commutant is much smaller than the weak commutant as we shall see in a moment. 
In order to motivate the considerations to follow let us take a look at a concrete example. Suppose $\mathscr{A}$ contains only one operator $A=i \frac{d}{d x}$ and the domain is $\mathscr{D}=\left\{f \in L_{2}\left(\left[0, \infty[) \mid f^{\prime} \in L_{2}([0, \infty[), f(0)=0\}\right.\right.\right.$ (scalar product defined by Lebesgue's measure). $A$ is a closed Hermitean operator with defect index $(0,1)$. It is easily seen that the strong commutant ${ }^{1}$ of $(\{A\}, \mathscr{D})$ is trivial. However, $A$ has many self-adjoint extensions which lead out of the Hilbert space $\mathscr{H}(\mathscr{D})$. For instance, we might take $\hat{A}=i \frac{d}{d x}$ on $\hat{\mathscr{D}}=\left\{f \in L_{2}(-\infty, \infty) \mid f^{\prime} \in L_{2}(-\infty, \infty)\right\}$ where $\mathscr{D}$ is identified with those functions in $\hat{\mathscr{D}}$ which vanish for $x \leqq 0$. Every such extension has a complete spectral decomposition $\hat{A}=\int \lambda d E_{\lambda}$, which defines at least a generalized decomposition of $A$ (cf. [6], Appendix I). For us, the important point is that this decomposition is associated with an abelian $*$-algebra in the strong commutant of the extension (in this case the whole strong commutant). This algebra will show up, somewhat distorted, in the weak commutant of $A$, namely if $b$ commutes strongly with $\hat{A}$ and $P$ is the projector $\mathscr{H}(\hat{\mathscr{D}}) \rightarrow \mathscr{H}(\mathscr{D})$, then $\mathrm{PbP}$ belongs to the weak commutant of $(\{A\}, \mathscr{D})$. The mapping $b \mapsto P b P$ will only in special cases preserve the operator product, so the image will not be an algebra. But as we are going to show, it is nevertheless possible to reverse this procedure and construct an extension of an operator family and an abelian algebra in the strong commutant of this extension, starting from certain subsets of the weak commutant of the family.

Since the mapping $b \mapsto P b P$ will occur repeatedly in the following we introduce a notation for it.

2.8. Definition. Let $(\hat{\mathscr{A}}, \hat{\mathscr{D}})$ be an extension of $(\mathscr{A}, \mathscr{D})$ and denote by $P$ the projector of $\mathscr{H}(\hat{\mathscr{D}})$ onto $\mathscr{H}(\mathscr{D})$. For any bounded operator $x$ on $\mathscr{H}(\hat{\mathscr{D}})$ the expression $P x \mid \mathscr{H}(\mathscr{D})$ defines a bounded operator on $\mathscr{H}(\mathscr{D})$. We denote this operator also by $\varrho(x)$.

The following properties of $\varrho$ are easily checked.

2.9. Lemma. (i) $\varrho$ is a linear mapping.

(ii) $\varrho(x) \leqq \varrho(y)$ if $x \leqq y$, so $\varrho\left(x^{*}\right)=\varrho(x)^{*}$.

(iii) $\varrho$ is weakly continuous.

(iv) If $x \in(\hat{\mathscr{A}}, \hat{\mathscr{D}})_{w}^{\prime}$ then $\varrho(x) \in(\mathscr{A}, \mathscr{D})_{w}^{\prime}$.

The concept of an extension without further restriction is too wide for our purposes because all possible extensions do not even form a set. Moreover, we are only interested in extensions which are rather closely linked to the weak commutant of the given operator family. In our construction of a suitable extension the following concepts will be relevant.

2.10. Definition. (1) A triple $(\hat{\mathscr{A}}, \hat{\mathscr{M}}, \hat{\mathscr{D}})$ will be called an induced extension of $(\mathscr{A}, \mathscr{D})$ if

(i) $(\hat{\mathscr{A}}, \hat{\mathscr{D}})$ is an extension of $(\mathscr{A}, \mathscr{D})$.

(ii) $\hat{\mathscr{M}}$ is a commutative *-algebra containing 1 and $\hat{\mathscr{M}} \subset(\hat{\mathscr{A}}, \hat{\mathscr{D}})_{s}^{\prime}$.

(iii) $\hat{\mathscr{D}}$ is the linear span of $\hat{\mathscr{M}} \mathscr{D}$.

(2) An induced extension $(\hat{\mathscr{A}}, \hat{\mathscr{M}}, \hat{\mathscr{D}})$ of $(\mathscr{A}, \mathscr{D})$ will be called regular, if the map $\varrho$ is one-to-one, when restricted to $(\hat{\mathscr{A}}, \hat{\mathscr{M}}, \hat{\mathscr{D}})_{w}^{\prime}:=(\hat{\mathscr{A}}, \hat{\mathscr{D}})_{w}^{\prime} \cap \hat{\mathscr{M}}^{\prime}$, i.e. $x \in(\hat{\mathscr{A}}, \hat{\mathscr{M}}, \hat{\mathscr{D}})_{w}^{\prime}$ and $\varrho(x)=0$ implies $x=0$.

\footnotetext{
1 More correctly: its Hermitean part.
} 
(3) Let $\hat{\mathscr{A}} \vee \hat{\mathscr{M}}$ denote the linear hull of $\hat{\mathscr{A}} \cup \hat{\mathscr{M}} \cup \hat{\mathscr{M}} \cdot \hat{\mathscr{A}}$. We define an order relation (transitive and reflexive) among the induced extensions of $(\mathscr{A}, \mathscr{D})$ as follows:

if there is a subalgebra $\hat{\mathscr{M}} \subset \hat{\hat{N}}$, such that

$$
(\hat{\mathscr{A}}, \hat{\mathscr{M}}, \hat{\mathscr{D}}) \prec(\hat{\mathscr{A}}, \hat{\hat{N}}, \hat{\mathscr{D}})
$$

$$
(\hat{\mathscr{A}} \vee \hat{\mathscr{M}}, \hat{\hat{N}}, \hat{\mathscr{D}}) \quad \text { is an induced extension of }(\hat{\mathscr{A}} \vee \hat{\mathscr{M}}, \hat{\mathscr{D}}) \text {. }
$$

Before we consider the existence of regular induced extensions we want to show that these concepts have reasonable properties.

2.11. Lemma. (i) If $(\hat{\mathscr{A}}, \hat{\mathscr{M}}, \hat{\mathscr{D}})$ is an induced extension of $(\mathscr{A}, \mathscr{D})$ and $B$ is a bounded operator in $\mathscr{A}$, then the equality $\|B\|=\|\hat{B}\|$ holds.

(ii) Let $(\hat{\mathscr{A}}, \hat{\mathscr{M}}, \hat{\mathscr{D}})$ be an induced extension. Then the weak closure $\hat{\mathscr{M}}^{-}$of $\hat{\mathscr{M}}$ defines an induced extension $\left(\hat{\mathscr{A}}^{-}, \hat{\mathscr{M}}^{-}, \hat{\mathscr{D}}^{-}\right) \succ(\hat{\mathscr{A}}, \hat{\mathscr{M}}, \hat{\mathscr{D}})$. If $(\hat{\mathscr{A}}, \hat{\mathscr{M}}, \hat{\mathscr{D}})$ is regular this extension is also regular.

(iii) Suppose $(\hat{\mathscr{A}}, \hat{\mathscr{M}}, \hat{\mathscr{D}})$ is a regular induced extension of $(\mathscr{A}, \mathscr{D})$, and $(\hat{\mathscr{A}} \vee \hat{\hat{M}}$, $\hat{\mathscr{L}}, \hat{\mathscr{D}})$ a regular induced extension of $(\hat{\mathscr{A}} \vee \hat{\mathscr{M}}, \hat{\mathscr{D}})$. Then $(\hat{\mathscr{A}}, \hat{\hat{N}}, \hat{\mathscr{D}})$ with $\hat{\hat{N}}=\hat{\hat{M}} \vee \hat{\mathscr{L}}$ is a regular induced extension of $(\mathscr{A}, \mathscr{D})$ and $(\hat{\mathscr{A}}, \hat{\mathscr{M}}, \hat{\mathscr{D}}) \prec(\hat{\mathscr{A}}, \hat{\hat{\mathscr{N}}}, \hat{\mathscr{D}})$.

(iv) Every linearily ordered (Definition 2.10 (iii)) family of regular induced extensions of $(\mathscr{A}, \mathscr{D})$ is majorized by a regular induced extension.

Proof. (i) We show that $B \geqq 0$ implies $\hat{B} \geqq 0$ for a bounded operator $B \in \mathscr{A}$. This means that upper and lower bounds are conserved by the extension which is sufficient for equality of the operator norms for Hermitean operators. Every vector in $\hat{\mathscr{D}}$ has the form $\sum_{i=1}^{n} m_{i} \varphi_{i}$ with $m_{i} \in \hat{\mathscr{M}}$ and $\varphi_{i} \in \mathscr{D}$. Since $\hat{\mathscr{M}}$ commutes strongly with $\hat{\mathscr{A}}$ and $\hat{\mathscr{A}} \hat{\imath} \mathscr{D}=\mathscr{A}$, we have for all $B \in \mathscr{A}$ :

$$
\begin{aligned}
\left\langle\sum_{i=1}^{n} m_{i} \varphi_{i}, \hat{B} \sum_{j=1}^{n} m_{j} \varphi_{j}\right\rangle & =\sum_{i j}\left\langle\varphi_{i},\left(m_{i}^{*} m_{j}\right) B \varphi_{j}\right\rangle \\
& =\sum_{i j}\left\langle\varphi_{i}, P\left(m_{i}^{*} m_{j}\right) P B \varphi_{j}\right\rangle
\end{aligned}
$$

where $P$ is the projector $\mathscr{H}(\hat{\mathscr{D}}) \rightarrow \mathscr{H}(\mathscr{D})$. If $B$ is bounded, it has a continuous extension to $\mathscr{H}(\mathscr{D})$ which commutes strongly with $P\left(m_{i}^{*} m_{j}\right) P$ because $B$ commutes weakly with this operator on $\mathscr{D}$. Therefore, if $B \geqq 0$. $B^{\frac{1}{2}}$ commutes strongly with $P\left(m_{i}^{*} \cdot m_{j}\right) P$ and

$$
\sum_{i j}\left\langle\varphi_{i}, P\left(m_{i}^{*} m_{j}\right) P B \varphi_{j}\right\rangle=\left\|\sum_{i} m_{i} B^{\frac{1}{2}} \varphi_{i}\right\| \geqq 0 .
$$

It follows that the norm is preserved for $B$ bounded and Hermitean. For general bounded $B$ we have then at least that $\hat{B}$ is bounded, and if we apply the result to $B^{*} B$, which has the extension $\hat{B} * \hat{B}$, we obtain $\|\hat{B}\|=\|B\|$.

(ii) The weak closure $\hat{\mathscr{M}}^{-}$commutes strongly with the closures $\hat{A}^{-}$of the operators $\hat{A} \in \hat{\mathscr{A}}$ according to Lemma 2.6 (vi). Therefore, $\hat{\mathscr{D}}^{-}$: = linear span $\hat{\mathscr{M}}^{-} \mathscr{D}=$ linear span $\hat{\mathscr{M}}^{-} \hat{\mathscr{D}}$ is in the domain of all $\hat{A}^{-}$. The corresponding extension $\left(\hat{\mathscr{A}}^{-}, \hat{\mathscr{M}}^{-}, \hat{\mathscr{D}}^{-}\right)$obviously majorizes $(\hat{\mathscr{A}}, \hat{\mathscr{M}}, \hat{\mathscr{D}})$, and if $\varrho$ is one-to-one when restricted to $(\hat{\mathscr{A}}, \hat{\mathscr{M}}, \hat{\mathscr{D}})_{w}^{\prime}$ then also on $\left(\hat{\mathscr{A}}^{-}, \hat{\mathscr{M}}^{-}, \hat{\mathscr{D}}^{-}\right)_{w}^{\prime} \subset(\hat{\mathscr{A}}, \hat{\mathscr{M}}, \hat{\mathscr{D}})_{w}^{\prime}$. 
(iii) By (i) we have that all operators in $\hat{\mathscr{M}}$ are bounded, so $\hat{\hat{N}}=\hat{\hat{M}} \vee \hat{\mathscr{L}}$ is a commutative $*$-algebra of bounded operators. $\hat{\mathscr{M}}$, and therefore $\hat{\hat{N}}$, commutes strongly with $\hat{\mathscr{A}}$, because $\hat{\mathscr{M}}$ commutes strongly with $\hat{\mathscr{A}}$, and all algebraic relation are conserved by the extension. Hence, $(\hat{\mathscr{A}}, \hat{\mathscr{M}}, \hat{\mathscr{D}})$ is an induced extension of $(\mathscr{A}, \mathscr{D})$. As for the regularity we note that the projector $\mathscr{H}(\hat{\mathscr{D}}) \rightarrow \mathscr{H}(\mathscr{D})$ factorizes into the projectors $\mathscr{H}(\hat{\mathscr{D}}) \rightarrow \mathscr{H}(\hat{\mathscr{D}})$ and $\mathscr{H}(\hat{\mathscr{D}}) \rightarrow \mathscr{H}(\mathscr{D})$. Under the associated mappings $\varrho$, the weak commutant $(\hat{\mathscr{A}}, \hat{\hat{N}}, \hat{\mathscr{D}})_{w}^{\prime}=(\hat{\mathscr{A}} \vee \hat{\mathscr{M}}, \hat{\mathscr{L}}, \hat{\mathscr{D}})_{w}^{\prime}$ is first mapped into $(\hat{\mathscr{A}} \vee \hat{\mathscr{M}}, \hat{\mathscr{D}})_{w}^{\prime}=(\hat{\mathscr{A}}, \hat{\mathscr{M}}, \hat{\mathscr{D}})_{w}^{\prime}$ and then into $(\mathscr{A}, \mathscr{D})_{w}^{\prime}$. Since both mappings are injective, $(\hat{\mathscr{A}}, \hat{\hat{N}}, \hat{\mathscr{D}})$ is regular. The relation $(\hat{\mathscr{A}}, \hat{\hat{N}}, \hat{\mathscr{D}}) \succ(\hat{\mathscr{A}}, \hat{\mathscr{M}}, \hat{\mathscr{D}})$ is obvious.

(iv) Let $\mathscr{I}$ be a linearily ordered index set, and $\left\{\left(\mathscr{A}^{\alpha}, \mathscr{M}^{\alpha}, \mathscr{D}^{\alpha}\right)\right\}_{\alpha \in \mathscr{I}}$ a family of induced extensions with $\left(\mathscr{A}^{\alpha}, \mathscr{M}^{\alpha}, \mathscr{D}^{\alpha}\right) \prec\left(\mathscr{A}^{\beta}, \mathscr{M}^{\beta}, \mathscr{D}^{\beta}\right)$ for $\alpha \leqq \beta$. Define $\hat{\mathscr{D}}=\bigcup_{\alpha \in \mathscr{I}} \mathscr{D}^{\alpha}$. $\hat{\mathscr{D}}$ is in a natural way a pre-Hilbert space, because the space $\mathscr{D}^{\alpha}$ are linearily ordered by inclusion and the norm on $\mathscr{D}^{\alpha} C \mathscr{D}^{\beta}$ is the same as the norm induced by $\mathscr{D}^{\beta}$. Moreover, it is consistent to define the extension of an operator $A \in \mathscr{A}$ to $\mathscr{D}$ by $\hat{A} \uparrow \mathscr{D}^{\alpha}=A^{\alpha}$, where $A^{\alpha}$ is the extension of $A$ to $\mathscr{D}^{\alpha}$. The algebra $\hat{\mathscr{M}}$ is defined as follows: For every $\mathscr{D}^{\alpha}$ we define $\hat{\mathscr{M}} \uparrow \mathscr{D}^{\alpha}=\bigcup_{\beta \geqq \alpha} \mathscr{M}^{\beta} \uparrow \mathscr{D}^{\alpha}$. Every $m \in \hat{\mathscr{M}}$ maps $\mathscr{D}^{\alpha}$ into some $\mathscr{D}^{\beta} \supset \mathscr{D}^{\alpha}$, so $\hat{\mathscr{M}}$ leaves $\hat{\mathscr{D}}$ invariant. Moreover, every $m \in \hat{\mathscr{M}}$ is a bounded operator: Indeed, if $m \in \hat{\mathscr{M}}$, then for some $\alpha$ we have $m \uparrow \mathscr{D}^{\alpha}=: m_{\alpha} \in \mathscr{M}^{\alpha}$, i.e. $m_{\alpha}$ is a bounded operator on $\mathscr{D}^{\alpha}$. The restriction $m \uparrow \mathscr{D}^{\beta}$ for $\beta \geqq \alpha$ is an extension of $m_{\alpha}$ because $\left(\mathscr{A}^{\alpha}, \mathscr{M}^{\alpha}, \mathscr{D}^{\alpha}\right) \prec\left(\mathscr{A}^{\beta}, \mathscr{M}^{\beta}, \mathscr{D}^{\beta}\right)$ implies in particular, that there is a subalgebra $\mathscr{M}^{\alpha \beta} \subset \mathscr{M}^{\beta}$ such that $\left(\mathscr{M}^{\alpha \beta}, \mathscr{M}^{\beta}, \mathscr{D}^{\beta}\right)$ is an induced extension of $\left(\mathscr{M}^{\alpha}, \mathscr{D}^{\alpha}\right)$. According to (i) an induced extension does not change the norm of an operator. Therefore, for $\beta \geqq \alpha$ we have that the norm of $m \uparrow \mathscr{D}^{\beta}$ is independent of $\beta$ so $m$ is a bounded operator on $\hat{\mathscr{D}}=\bigcup_{\beta \geqq \alpha} \mathscr{D}^{\beta}$.

It is obvious that $\hat{\mathscr{D}}=\stackrel{\beta \geqq \alpha}{=}$ linear span $\hat{\mathscr{M}} \mathscr{D}$ and $(\hat{\mathscr{A}}, \hat{\mathscr{M}}, \hat{\mathscr{D}}) \succ\left(\mathscr{A}^{\alpha}, \mathscr{M}^{\alpha}, \mathscr{D}^{\alpha}\right)$ for every $\alpha \in \mathscr{I}$.

It remains to show that the extension $(\hat{\mathscr{A}}, \hat{\mathscr{M}}, \hat{\mathscr{D}})$ is regular if this is the case for all $\left(\mathscr{A}^{\alpha}, \mathscr{M}^{\alpha}, \mathscr{D}^{\alpha}\right)$. Let $P_{0}, P_{0 \alpha}$ and $P_{\alpha}$ denote the projectors $\mathscr{H}(\mathscr{D}) \rightarrow \mathscr{H}(\mathscr{D})$, $\mathscr{H}\left(\mathscr{D}^{\alpha}\right) \rightarrow \mathscr{H}(\mathscr{D})$ and $\mathscr{H}(\hat{\mathscr{D}}) \rightarrow \mathscr{H}\left(\mathscr{D}^{\alpha}\right)$ respectively. Suppose $x \in(\hat{\mathscr{A}}, \hat{\mathscr{M}}, \hat{\mathscr{D}})_{w}^{\prime}$ and $P_{0} \times P_{0}=0$. Because $P_{0}=P_{0 \alpha} \cdot P_{\alpha}$ and $P_{\alpha} \times P_{\alpha} \in\left(\mathscr{A}^{\alpha}, \mathscr{M}^{\alpha}, \mathscr{D}^{\alpha}\right)_{w}^{\prime}$, the regularity of $\left(\mathscr{A}^{\alpha}, \mathscr{M}^{\alpha}, \mathscr{D}^{\alpha}\right)$ implies $P_{\alpha} \times P_{\alpha}=0$. Hence, if all $\left(\mathscr{A}^{\alpha}, \mathscr{M}^{\alpha}, \mathscr{D}^{\alpha}\right)$ are regular, the matrix elements of $x$ vanish for a dense set of vectors which means that $x=0$. Therefore $(\hat{\mathscr{A}}, \hat{\mathscr{M}}, \hat{\mathscr{D}})$ is regular.

At this point we would like to outline the procedure in the remaining part of this section and comment on the role of regularity as defined in 2.10 . What we are looking for is an induced extension $(\hat{\mathscr{A}}, \hat{\mathscr{M}}, \hat{\mathscr{D}})$ with the property that the weak and the strong commutant of $(\hat{\mathscr{A}} \vee \hat{\mathscr{M}}, \hat{\mathscr{D}})$ are identical and equal to $\hat{\mathscr{M}}$. For the construction of induced extensions we use a simple device by which any element $x \in(\mathscr{A}, \mathscr{D})_{w}^{\prime}$ with $0 \leqq x \leqq 1$ can be "lifted" to an element commuting strongly with an extension defined by $x$. In general it will be necessary to repeat this construction an infinite number of times, and this means that we must appeal to Zorn's lemma to guarantee that we shall eventually end up with some maximal extension. We are then led to the problem of specifying a set of extensions on 
which Zorn's lemma can be applied. For this purpose it is natural to consider induced extensions for which the restriction $\varrho \uparrow \hat{\mathscr{M}}$ is one-to-one, because these extensions are essentially determined by the subsets $\varrho(\mathscr{M}) \subset(\mathscr{A}, \mathscr{D})_{w}^{\prime}$ as we are going to show presently. Having constructed any such "nice" extension $(\hat{\mathscr{A}}, \hat{\mathscr{M}}, \hat{\mathscr{D}})$ we cannot expect it to be maximal and shall usually have to proceed further and construct a "nice" extension of this extension, say $(\hat{\mathscr{A}} \vee \hat{\mathscr{M}}, \hat{\mathscr{N}}, \hat{\mathscr{D}})$. Now the corresponding $\varrho$ will map $\hat{\mathscr{N}}$ into $(\hat{\mathscr{A}}, \hat{\mathscr{M}}, \hat{\mathscr{D}})_{w}^{\prime}$. Therefore, in order to guarantee that a "nice" extension of a "nice" extension defines a "nice" extension of the original family $(\mathscr{A}, \mathscr{D})$, we require that the $\varrho$ corresponding to $(\hat{\mathscr{A}}, \hat{\mathscr{M}}, \hat{\mathscr{D}})$ is oneto-one not only on $\hat{\mathscr{M}}$ but on the larger set $(\hat{\mathscr{A}}, \hat{\mathscr{M}}, \hat{\mathscr{D}})_{w}^{\prime}$. This is the condition of regularity. Lemma 2.11 combined with Zorn's lemma will imply the existence of maximal regular extensions, and the remaining problem is essentially to find out which elements of the weak commutant define regular extensions and show that $(\hat{\mathscr{A}}, \hat{\mathscr{M}}, \hat{\mathscr{D}})$ is maximal if and only if $\hat{\mathscr{M}}=(\hat{\mathscr{A}}, \hat{\mathscr{M}}, \hat{D})_{w}^{\prime}$. Before we turn to this problem we want to establish the relation between induced extensions and the weak commutant $(\mathscr{A}, \mathscr{D})_{w}^{\prime}$.

2.12. Lemma. 1) Suppose $(\hat{\mathscr{A}}, \hat{\mathscr{M}}, \hat{D})$ is an induced extension of $(\mathscr{A}, \mathscr{D})$ with the property that $\varrho$ is one-to-one when restricted to $\hat{\mathscr{M}}$. Denote by $\mathscr{M}$ the set $\varrho(\hat{M})$ $C(\mathscr{A}, \mathscr{D})_{w}^{\prime}$ and define a map $\Phi: \mathscr{M} \times \mathscr{M} \rightarrow \mathscr{M}$ by

$$
\Phi\left(m_{1}, m_{2}\right)=\varrho\left(\varrho^{-1}\left(m_{1}\right) \cdot \varrho^{-1}\left(m_{2}\right)\right) .
$$

Let $\mathscr{K} \subset \mathscr{M}$ denote the set $\varrho\left(\hat{\mathscr{M}}^{+}\right)$, where $\hat{\mathscr{M}}^{+}$is the positive cone in $\mathscr{M}$.

Then $\mathscr{M}, \mathscr{K}$ and $\Phi$ have the following properties:

(i) $\mathscr{M}$ is a self-adjoint subspace of $(\mathscr{A}, \mathscr{D})_{w}^{\prime}$

(ii) $\mathscr{K}$ is a cone in $\mathscr{M}$ such that

(a) $\mathscr{K}$ generates $\mathscr{M}$ as a vector space.

(b) $\mathscr{K}$ contains the unit operator 1.

(c) If $m \in \mathscr{K}$, then there is a real number $0 \leqq \lambda(m)<\infty$ such that $\lambda(m) 1-m \in \mathscr{K}$.

(iii) $\Phi$ is a bilinear map $\mathscr{M} \times \mathscr{M} \rightarrow \mathscr{M}$ satisfying

(a) $\Phi\left(m_{1}, \Phi\left(m_{2}, m_{3}\right)\right)=\Phi\left(\Phi\left(m_{1}, m_{2}\right), m_{3}\right)=: \Phi\left(m_{1}, m_{2}, m_{3}\right)$ (associativity).

(b) $\Phi\left(m_{1}, m_{2}\right)=\Phi\left(m_{2}, m_{1}\right) \quad$ (symmetry).

(c) $\Phi(m, 1)=m \quad$ (unit element).

(d) $\Phi(m, n)^{*}=\Phi\left(m^{*}, n^{*}\right)$.

(e) $\Phi$ is positive on $\mathscr{K}$ in the sense that for all $m \in \mathscr{K}$ and all finite collections of $m_{i} \in \mathscr{M}, \varphi_{i} \in \mathscr{D}$ we have

$$
\sum_{i j}\left\langle\varphi_{i}, \Phi\left(m_{i}^{*}, m, m_{j}\right) \varphi_{j}\right\rangle \geqq 0 .
$$

2) Conversely, let $\mathscr{M}, \mathscr{K}$ and $\Phi$ satisfy the conditions (i)-(iii). Then there is an induced extension $(\hat{\mathscr{A}}, \hat{\mathscr{M}}, \hat{\mathscr{D}})$ of $(\mathscr{A}, \mathscr{D})$ such that $\varrho \uparrow \hat{\mathscr{M}}$ is one-to-one, and

(a) $\mathscr{M}=\varrho(\hat{M})$.

(b) $\Phi\left(m_{1}, m_{2}\right)=\varrho\left(\varrho^{-1}\left(m_{1}\right) \cdot \varrho^{-1}\left(m_{2}\right)\right)$.

(c) $\mathscr{K} \subset \varrho\left(\hat{\mathscr{M}}^{+}\right)$.

This extension is uniquely determined by (a) and (b), up to unitary equivalence. Moreover, if $\left(\mathscr{\mathscr { A }}^{1}, \hat{\mathscr{M}}^{1}, \hat{\mathscr{D}}^{1}\right)$ and $\left(\hat{\mathscr{A}}^{2}, \hat{\mathscr{M}}^{2}, \hat{\mathscr{D}}^{2}\right)$ are two extensions associated with 
$\left(\mathscr{M}^{1}, \mathscr{K}^{1}, \Phi^{1}\right)$ resp. $\left(\mathscr{M}^{2}, \mathscr{K}^{2}, \Phi^{2}\right)$, then the relation

$$
\left(\hat{\mathscr{A}}^{1}, \hat{\mathscr{M}}^{1}, \hat{\mathscr{D}}^{1}\right) \prec\left(\hat{\mathscr{A}}^{2}, \hat{\mathscr{M}}^{2}, \hat{\mathscr{D}}^{2}\right)
$$

holds if and only if $\mathscr{M}^{1} \subset \mathscr{M}^{2}$ and $\Phi^{1}=\Phi^{2} \uparrow \mathscr{M}^{1} \times \mathscr{M}^{1}$.

Proof. 1) The first statement (i) follows from Lemma 2.9. Since $\hat{\mathscr{M}}^{+}$generates $\hat{\mathscr{M}}$ we have also (ii) (a), and (b) follows because $1=\varrho(\hat{1})$, where $\hat{1} \in \hat{\mathscr{M}}$ is the unit operator on $\mathscr{H}(\hat{\mathscr{D}})$. To verify (ii) (c) we note that $\|\hat{m}\| \hat{1}-\hat{m} \in \hat{\mathscr{M}}^{+}$for all $\hat{m} \in \hat{\mathscr{M}}^{+}$. Therefore, if $m \in \mathscr{K}$, then $\left\|\varrho^{-1}(m)\right\| 1-m \in \mathscr{K}$.

The properties (iii) (a)-(c) follow immediately from the definition of $\Phi$. For (iii) (d) note that $\varrho$ preserves adjoints and is one-to-one on $\hat{\mathscr{M}}$. Therefore we have also $\varrho^{-1}\left(m^{*}\right)=\varrho^{-1}(m)^{*}$ from which the assertion follows. Finally, $\varrho(x)=P \times P$ by definition, so if $m \in \mathscr{K}$ we have

$$
\sum_{i j}\left\langle\varphi_{i}, \Phi\left(m_{i}^{*}, m, m_{j}\right) \varphi_{j}\right\rangle=\left\langle\sum_{i} \varrho^{-1}\left(m_{i}\right) \varphi_{i}, \varrho^{-1}(m) \sum_{j} \varrho^{-1}\left(m_{j}\right) \varphi_{j}\right\rangle \geqq 0
$$

because $\varrho^{-1}(m) \in \hat{\mathscr{M}}^{+}$.

(2) Let us consider the uniqueness of the extension first. Suppose $\left(\hat{\mathscr{A}}^{1}, \hat{\mathscr{M}}^{1}, \hat{\mathscr{D}}^{1}\right)$ and $\left(\hat{\mathscr{A}}^{2}, \hat{\mathscr{M}}^{2}, \hat{\mathscr{D}}^{2}\right)$ are two extensions with corresponding mappings $\varrho^{1}$ resp. $\varrho^{2}$ such that

$(\alpha) \varrho^{1 \uparrow} \hat{\mathscr{M}}^{1}$ resp. $\varrho^{2} \uparrow \hat{\mathscr{M}}^{2}$ is one-to-one.

(B) $\varrho^{1}\left(\hat{\mathscr{M}}^{1}\right)=\varrho^{2}\left(\hat{\mathscr{M}}^{2}\right)=: \mathscr{M}$.

$(\gamma) \varrho^{1}\left(\varrho^{1^{-1}}\left(m_{1}\right) \cdot \varrho^{1^{-1}}\left(m_{2}\right)\right)=\varrho^{2}\left(\varrho^{2^{-1}}\left(m_{1}\right) \cdot \varrho^{2-1}\left(m_{2}\right)\right)$ for all $m_{1}, m_{2} \in \mathscr{M}$.

We define a linear operator $U: \hat{\mathscr{D}}^{1} \rightarrow \mathscr{D}^{2}$ as follows:

$$
U\left(\sum_{i} \hat{m}_{i} \varphi_{i}\right)=\sum_{i} \varrho^{2^{-1}} \cdot \varrho^{1}\left(\hat{m}_{i}\right) \varphi_{i}
$$

for $\hat{m}_{i} \in \hat{\mathscr{M}}^{1}, \varphi_{i} \in \mathscr{D}$

One checks easily that $U$ is a well defined isometric operator which can be extended to a unitary operator $\mathscr{H}\left(\hat{\mathscr{D}}^{1}\right) \rightarrow \mathscr{H}\left(\hat{\mathscr{D}}^{2}\right)$ and that $U \hat{\mathscr{M}}^{1} U^{-1}=\hat{\mathscr{M}}^{2}$, $U \hat{A}^{1} U^{-1}=\hat{A}^{2}$ for all $A \in \mathscr{A}$, and $U$ is the identity when restricted to $\mathscr{H}(\mathscr{D})$.

The reconstruction of an extension corresponding to given $\mathscr{M}, \mathscr{K}$, and $\Phi$ is carried out in a more or less canonical fashion: We equip the algebraic tensor product $\mathscr{M} \otimes \mathscr{H}(\mathscr{D})$ with an inner product by defining

$$
\left\langle\sum_{i} m_{i} \otimes \varphi_{i}, \sum_{j} n_{j} \otimes \psi_{j}\right\rangle=\sum_{i j}\left\langle\varphi_{i}, \Phi\left(m_{i}^{*}, n_{j}\right) \psi_{j}\right\rangle .
$$

This product is nonnegative because of condition (iii) (e).

We form the quotient space with respect to vectors of zero length and define $\hat{\mathscr{D}}$ as the image of $\mathscr{M} \otimes \mathscr{D}$. $\mathscr{D}$ can be identified with (the image of) $1 \otimes \mathscr{D}$. If $\left[\sum_{i} m_{i} \otimes \varphi_{i}\right] \in \hat{\mathscr{D}}$ is a rest class corresponding to $\sum_{i} m_{i} \otimes \varphi_{i} \in \mathscr{M} \otimes \mathscr{D}$ and $A \in \mathscr{A}$, we define

$$
\hat{A}\left[\sum_{i} m_{i} \otimes \varphi_{i}\right]=\left[\sum_{i} m_{i} \otimes A \varphi_{i}\right] .
$$


This is well defined because $\mathscr{M}$ commutes weakly with $A$ : Indeed, if $\left[\sum_{i} m_{i} \otimes \varphi_{i}\right]=0$, then we have for all $n \otimes \psi$ :

$$
\begin{aligned}
\left\langle n \otimes \psi, \sum_{i} m_{i} \otimes A \varphi_{i}\right\rangle & =\left\langle\psi, \sum_{i} \Phi\left(n^{*}, m_{i}\right) A \varphi_{i}\right\rangle \\
& =\left\langle A^{*} \psi, \sum_{i} \Phi\left(n^{*}, m_{i}\right) \varphi_{i}\right\rangle=\left\langle n \otimes A^{*} \psi, \sum_{i} m_{i} \otimes \varphi_{i}\right\rangle
\end{aligned}
$$

$=0$ by Cauchy-Schwarz, so $\left[\sum_{i} m_{i} \otimes A \varphi_{i}\right]=0$.

The algebra $\hat{\mathscr{M}}$ is defined as follows. For $m \in \mathscr{M}$ define

$$
\hat{m}\left[\sum_{i} m_{i} \otimes \varphi_{i}\right]=\left[\sum_{i} \Phi\left(m, m_{i}\right) \otimes \varphi_{i}\right] .
$$

The associativity of $\Phi$ implies that $\hat{m}$ is well defined:

$$
\begin{aligned}
\left\langle n \otimes \psi, \sum_{i} \Phi\left(m, m_{i}\right) \otimes \varphi_{i}\right\rangle & =\left\langle\psi, \sum_{i} \Phi\left(n^{*}, m, m_{i}\right) \varphi_{i}\right\rangle \\
& =\left\langle\Phi\left(n^{*}, m\right)^{*} \otimes \psi, \sum_{i} m_{i} \otimes \varphi_{i}\right\rangle
\end{aligned}
$$

so if $\left[\sum_{i} m_{i} \otimes \varphi_{i}\right]=0$, then also $\left[\sum_{i} \Phi\left(m, m_{i}\right) \otimes \varphi_{i}\right]=0$.

Next we show that every $\hat{m}$ is a bounded operator. It is enough to do this for $m \in \mathscr{K}$ because the mapping $m \rightarrow \hat{m}$ is linear and $\mathscr{K}$ generates $\mathscr{M}$. But the positivity condition (iii) (e) yields $0 \leqq \hat{m}$ and by (ii) (c) also $0 \leqq \lambda(m) \hat{1}-\hat{m}$, so $\hat{m}$ is a bounded operator and can be extended to a bounded operator on $\mathscr{H}(\hat{\mathscr{D}})$.

From the definition of $\hat{m}$ and $\hat{A}$ it is clear that $\hat{m}$ maps $\hat{\mathscr{D}}$ into $\hat{\mathscr{D}}$ and commutes there strongly with every $\hat{A}$. Furthermore, from the symmetry of $\Phi$ it follows that different $\hat{m}$ 's commute. The invariance of $\hat{\mathscr{M}}$ under the $*$-operation follows from (iii) (d). Finally, the equation $m=\varrho(\hat{m})$ follows from

$$
\langle[1 \otimes \psi], \hat{m}[1 \otimes \varphi]\rangle=\langle\psi, \Phi(1, m, 1) \varphi\rangle=\langle\psi, m \varphi\rangle .
$$

We have thus constructed an induced extension satisfying the conditions (a), (b), and (c).

The verification of the last statement concerning different $\mathscr{M}$ 's and $\Phi$ 's is straightforward.

2.13. Lemma. Let $x$ be an element of $(\mathscr{A}, \mathscr{D})_{w}^{\prime}$ with $0 \leqq x \leqq 1$ and $x \neq \lambda 1$, $0<\lambda<1$. If $\mathscr{M}=\{\lambda x+\mu(1-x) \mid \lambda, \mu \in \mathbb{C}\}, \mathscr{K}=\{\lambda x+\mu(1-x) \mid \lambda, \mu \geqq 0\}$ and $\Phi$ is defined by

$$
\Phi(\lambda x+\mu(1-x), x x+v(1-x))=\lambda x x+\mu v(1-x),
$$

then all the conditions of Lemma 2.12 are satisfied, and $\hat{\mathscr{M}}$ is generated by $\hat{1}$ and a projector $e$ with $\varrho(e)=x$. If moreover $\|x\|=\|1-x\|=1$, then $\mathscr{K}$ is identical to $\{y \mid y \in \mathscr{M}, y \geqq 0\}$.

Proof. The conditions (i)-(iii) are all immediately verified. By construction, the algebra $\hat{\mathscr{M}}$ is generated by $\hat{1}$ and $\hat{x}=: e$. Since $x \in \mathscr{K}$ we have $e \geqq 0$, and $\Phi(x, x)=x$ implies $e^{2}=e$, so $e$ is a Hermitean projector.

If $\lambda x+\mu(1-x) \geqq 0$ and $\|x\|=\|1-x\|=1$, we show that $\lambda \geqq 0$ and $\mu \geqq 0$ by taking supremum resp. infimum of $\langle\varphi,(\lambda x+\mu(1-x)) \varphi\rangle$ over all $\varphi$ with $\|\varphi\|=1$. 
If $\mathscr{M}, \mathscr{K}^{\prime}, \Phi$ are as in Lemma 2.13, we shall refer to the corresponding induced extension as the extension defined by $x$. The main step required for Theorem 2.16 is a characterization of those $x$ which define a regular induced extension.

2.14. Lemma. Let $x$ be as in Lemma 2.13. The extension defined by $x$ is regular if and only if $x$ is an extremal element of the weakly compact set

$$
\mathscr{W}_{1}^{+}:=\left\{y \in(\mathscr{A}, \mathscr{D})_{w}^{\prime} \mid 0 \leqq y \leqq 1\right\} .
$$

Moreover, if $x$ is extremal and $x \neq 0, x \neq 1$, then we have $\|x\|=\|1-x\|=1$.

The proof of this result depends on

2.15. Lemma. Let $x$ be as in Lemma 2.13 , denote by $\mathscr{W}_{x}$ the set

$$
\left\{y \in(\mathscr{A}, \mathscr{D})_{w}^{\prime} \mid \text { there is an } \varepsilon \in \mathbb{R}, \varepsilon \neq 0 \text { such that }-x \leqq \varepsilon y \leqq x\right\}
$$

and define $\mathscr{W}_{1-x}$ similarily.

The extension defined by $x$ is regular if and only if $\mathscr{W}_{x} \cap \mathscr{W}_{1-x}=\{0\}$.

Proof of Lemma 2.15. Let $e$ be the projector as in Lemma 2.13 and denote by $\hat{\mathscr{W}}$ the Hermitean part of $(\hat{\mathscr{A}}, \hat{\mathscr{M}}, \hat{\mathscr{D}})_{w}^{\prime}=(\hat{\mathscr{A}}, \hat{\mathscr{D}})_{w}^{\prime} \cap\{e\}^{\prime}$. We want to show that $\mathscr{W}_{x}=\varrho(e \hat{\mathscr{W}})$ and $\mathscr{W}_{1-x}=\varrho((1-e) \hat{\mathscr{W}})$. This will then quickly lead to the desired result.

Suppose $y \in \hat{\mathscr{W}}$. Since $e \in(\hat{\mathscr{A}}, \hat{\mathscr{D}})_{s}^{\prime} \cap\{e\}^{\prime}$ we have $e y=y e \in \hat{\mathscr{W}}$. Therefore $\varrho(e y)$ is Hermitean and belongs to $(\mathscr{A}, \mathscr{D})_{w}^{\prime}$. Moreover, because $e$ and $y$ commute, we have also

$$
-e \leqq \frac{e y}{\|y\|} \leqq e \text { and thus }-x \leqq \frac{\varrho(e y)}{\|y\|} \leqq x,
$$

so $\varrho(e y) \in \mathscr{W}_{x}$. Analogously one proves $\varrho((1-e) y) \in \mathscr{W}_{1-x}$. This shows that $\varrho(e \hat{\mathscr{W}}) \subset \mathscr{W}_{x}$ and $\varrho((1-e) \hat{\mathscr{W}}) \subset \mathscr{W}_{1-x}$.

To get the other inclusion we note that $\hat{\mathscr{D}}=$ linear span $\hat{\mathscr{M}} \mathscr{D}=e \mathscr{D} \oplus(1-e) \mathscr{D}$. If $w \in \mathscr{W}_{x}$ we define an operator $\hat{w}$ by its matrix elements as follows:

$$
\left\langle e \varphi_{1}+(1-e) \psi_{1}, \hat{w}\left(e \varphi_{2}+(1-e) \psi_{2}\right)\right\rangle=\left\langle e \varphi_{1}, \hat{w} e \varphi_{2}\right\rangle:=\left\langle\varphi_{1}, w \varphi_{2}\right\rangle .
$$

This is well defined if we show that $\left\langle\varphi_{1}, w \varphi_{2}\right\rangle$ only depends on $e \varphi_{1}$ and $e \varphi_{2}$. By assumption, $w \in \mathscr{W}_{x}$, so $0 \leqq w+\lambda x \leqq 2 \lambda x$ for some $\lambda$. By definition of $e$ (cf. Lemma 2.13), we have $\|e \varphi\|=\langle\varphi, x \varphi\rangle^{\frac{1}{2}}$ for all $\varphi$. Hence, we conclude from

that

$$
\begin{aligned}
\left|\left\langle\varphi_{1}, w \varphi_{2}\right\rangle+\lambda\left\langle\varphi_{1}, x \varphi_{2}\right\rangle\right| \leqq\left\langle\varphi_{1},(w+\lambda x) \varphi_{1}\right\rangle^{\frac{1}{2}} & \\
\cdot & \left\langle\varphi_{2},(w+\lambda x) \varphi_{2}\right\rangle^{\frac{1}{2}} \leqq 2 \lambda\left\langle\varphi_{1}, x \varphi_{1}\right\rangle^{\frac{1}{2}} \cdot\left\langle\varphi_{2}, x \varphi_{2}\right\rangle^{\frac{1}{2}}
\end{aligned}
$$

$$
\left|\left\langle\varphi_{1}, w \varphi_{2}\right\rangle\right| \leqq 3 \lambda\left\langle\varphi_{1}, x \varphi_{1}\right\rangle^{\frac{1}{2}} \cdot\left\langle\varphi_{2}, x \varphi_{2}\right\rangle^{\frac{1}{2}}=3 \lambda\left\|e \varphi_{1}\right\| \cdot\left\|e \varphi_{2}\right\|
$$

so $\hat{w}$ is a well defined bounded operator. It is obvious that $\hat{w}=e \hat{w}$ and $\varrho(\hat{w})=w$. Similarly one shows that $\varrho((1-e) \hat{\mathscr{W}})=\mathscr{W}_{1-x}$.

We can now prove, that if the extension is regular, then we have $\mathscr{W}_{x} \cap \mathscr{W}_{1-x}$ $=\{0\}$. Indeed, assume that $w \in \mathscr{W}_{x} \cap \mathscr{W}_{1-x}, w \neq 0$. Then we find elements $e \hat{w}_{1} \in e \mathscr{W}$ and $(1-e) \hat{w}_{2} \in(1-e) \hat{\mathscr{W}}$ with $\varrho\left(e \hat{w}_{1}-(1-e) \hat{w}_{2}\right)=w-w=0$. Since $e \hat{w}_{1}$ and $(1-e) \hat{w}_{2}$ belong to orthogonal subspaces, we have $\hat{w}=e \hat{w}_{1}-(1-e) \hat{w}_{2} \neq 0$, so the extension is not regular. 
On the other hand, the matrix elements of an operator $y \in \hat{\mathscr{W}}$ are

$$
\begin{aligned}
\left\langle e \varphi_{1}+(1-e) \psi_{2}, y\left(e \varphi_{2}\right.\right. & \left.\left.+(1-e) \psi_{2}\right)\right\rangle \\
= & \left\langle\varphi_{1}, \varrho(e y) \varphi_{2}\right\rangle+\left\langle\psi_{1}, \varrho((1-e) y) \psi_{2}\right\rangle
\end{aligned}
$$

with $\varphi_{i}, \psi_{i} \in \mathscr{D}$. If $\mathscr{W}_{x} \cap \mathscr{W}_{1-x}=\{0\}$, then it follows from $0=\varrho(y)=\varrho(e y)+\varrho((1-e) y)$ that $\varrho(e y)=\varrho((1-e) y)=0$. By the formula above, this implies $y=0$, so the extension is regular.

Proof of Lemma 2.14. By definition, $x$ is an extreme point in $\mathscr{W}_{1}^{+}$if and only if $x=\frac{1}{2} x_{1}+\frac{1}{2} x_{2}, x_{i} \in \mathscr{W}_{1}^{+}$, implies $x_{1}=x_{2}=x$. Because $\frac{1}{2} x_{1}+\frac{1}{2} x_{2}=\frac{1}{2}(x-y)+\frac{1}{2}(x+y)$ with $y=x-x_{1}=x_{2}-x$, this can also be stated as follows: $x$ is extreme iff $x+y$ and $x-y \in \mathscr{W}_{1}^{+}$implies $y=0$. But $0 \leqq x+y \leqq 1$ and $0 \leqq x-y \leqq 1$ is equivalent to $-x \leqq y \leqq 1-x$ and $-(1-x) \leqq y \leqq x$ which in turn means $-x \leqq y \leqq x$ and $-(1-x) \leqq y \leqq(1-x)$. Thus, $y=0$ if and only if $\mathscr{W}_{x} \cap \mathscr{W}_{1-x}=\{0\}$

It remains to show that if $x$ is extreme and $x \neq 0,1$, then we have $\|x\|$ $=\|1-x\|=1$. Since clearly $x$ is extreme, iff $1-x$ is extreme, it is enough to prove that $x$ is extreme and $x \neq 0$ implies $\|x\|=1$. This follows from the formula

$$
x=\lambda \frac{x}{\|x\|}+(1-\lambda)\|x\| x
$$

with $\lambda=\frac{\|x\|-\|x\|^{2}}{1-\|x\|^{2}}$, which yields a nontrivial decomposition of $x$ if $\|x\|<1$.

We are now prepared for the main result of this section.

2.16. Theorem. (1) Every regular induced extension of $(\mathscr{A}, \mathscr{D})$ is majorized by a maximal regular induced extension.

(2) A regular induced extension $(\hat{\mathscr{A}}, \hat{\mathscr{M}}, \hat{\mathscr{D}})$ is maximal if and only if

$$
(\hat{\mathscr{A}}, \hat{\mathscr{M}}, \hat{\mathscr{D}})_{w}^{\prime}=(\hat{\mathscr{A}}, \hat{\mathscr{M}}, \hat{\mathscr{D}})_{s}^{\prime}=\hat{\mathscr{M}}
$$

(3) To every extremal element $x$ in $\mathscr{W}_{1}^{+}$there exists a maximal regular induced extension such that $x$ is the image under $\varrho$ of a projector in $\hat{\mathscr{M}}$.

Proof. (1) We define unitary equivalence of induced extensions in an obvious manner, which was already implicit in Lemma 2.12(2): Two extensions $\left(\hat{\mathscr{A}}^{1}, \hat{\mathscr{M}}^{1}, \hat{\mathscr{D}}^{1}\right)$ and $\left(\hat{\mathscr{A}}^{2}, \hat{\mathscr{M}}^{2}, \hat{\mathscr{D}}^{2}\right)$ are unitarily equivalent if there is a unitary operator $U: \mathscr{H}\left(\hat{\mathscr{D}}^{1}\right) \rightarrow \mathscr{H}\left(\hat{\mathscr{D}}^{2}\right)$ such that $U \uparrow \mathscr{H}(\mathscr{D})$ is the identity, $U \hat{\mathscr{M}}^{1} U^{-1}=\hat{\mathscr{M}}^{2}$ and $U \hat{A}^{1} U^{-1}=\hat{A}^{2}$ for all $A \in \mathscr{A}$. By Lemma 2.12 the equivalence classes of regular extensions form a set because they are in a one-to-one correspondence with certain subsets of $(\mathscr{A}, \mathscr{D})_{w}^{\prime}$ and bilinear maps on there sets. The order relation for extensions defines in a canonical way an order relation on the set of equivalence classes of regular extensions because of the last statement of Lemma 2.12 (2). By Lemma 2.11 (iv), this set is inductive (and not empty, because $(\mathscr{A},\{\lambda 1\}, \mathscr{D})$ is a trivial extension) so the assertion follows from Zorn's lemma.

(2) Suppose $(\hat{\mathscr{A}}, \hat{\mathscr{M}}, \hat{\mathscr{D}})$ is a maximal regular extension. If $\hat{\mathscr{M}}$ is a proper subset of $(\hat{\mathscr{A}}, \hat{\mathscr{M}}, \hat{\mathscr{D}})_{w}^{\prime}=(\hat{\mathscr{A}} \vee \hat{\mathscr{M}}, \hat{\mathscr{D}})_{w}^{\prime}$, then we have also that the Hermitean part of $\hat{\mathscr{M}}$ is a proper subset of the Hermitean part of $(\hat{\mathscr{A}} \vee \hat{\mathscr{M}}, \hat{\mathscr{D}})_{w}^{\prime}$. Therefore, $\hat{\mathscr{M}}_{1}^{+}:=\{y \in \mathscr{M} \mid 0 \leqq y \leqq 1\}$ is a proper subset of $\hat{\mathscr{W}}_{1}^{+}=\left\{y \in(\mathscr{A} \vee \mathscr{M}, \mathscr{D})_{w}^{\prime} \mid 0 \leqq y \leqq 1\right\}$. 
By Lemma 2.11 (ii) we have that $\hat{\mathscr{M}}$ and therefore $\hat{\mathscr{M}}_{1}^{+}$is weakly closed. Since $\hat{\mathscr{M}}_{1}^{+}$is moreover convex, we conclude from the Krein-Milman theorem, applied to the convex, compact set $\hat{\mathscr{W}}_{1}^{+}$, that there exists an extremal element $x \in \hat{\mathscr{W}}_{1}^{+}$with $x \notin \hat{\mathscr{M}}_{1}^{+}$. Since $\hat{\mathscr{M}}_{1}^{+}=\hat{\mathscr{M}} \cap \hat{\mathscr{W}}_{1}^{+}$, this $x$ does not belong to $\hat{\mathscr{M}}$ and by Lemma 2.14 it defines a regular induced extension of $(\hat{\mathscr{A}} \vee \mathscr{M}, \hat{\mathscr{D}})$. This contradicts the maximality of $(\hat{\mathscr{A}}, \hat{\mathscr{M}}, \hat{\mathscr{D}})$ by Lemma 2.11 (iii).

(3) By Lemma 2.14, every extremal element $x$ defines a regular extension $(\hat{\mathscr{A}}, \hat{\mathscr{M}}, \hat{\mathscr{D}})$ of $(\mathscr{A}, \mathscr{D})$, such that $x=\varrho(e)$ with a projector $e \in \hat{\mathscr{M}}$. Let $(\hat{\mathscr{A}}, \hat{\mathscr{N}}, \hat{\mathscr{D}})$ be a maximal regular extension majorizing $(\hat{\mathscr{A}}, \hat{\mathscr{M}}, \hat{\mathscr{D}})$. We have to show that the extension $\hat{e}$ of $e$ to $\hat{\mathscr{D}}$ is also a projector. If $\hat{\hat{n}} \varphi \in \hat{\mathscr{D}}$ with $\hat{\hat{n}} \in \hat{\mathscr{N}}, \varphi \in \mathscr{D}$, then we have $\hat{e}^{2} \hat{\hat{n}} \varphi=\hat{\hat{n}} e^{2} \varphi=\hat{n} e \varphi=\hat{e} \hat{n} \varphi$, so $\hat{e}^{2}=\hat{e}$ and $\hat{e}$ is a projector.

\section{Decomposition Theory}

In this section we shall impose some further conditions on $\mathscr{A}$ and $\mathscr{D}$ under which we can prove that a decomposition of the abelian algebra $\hat{\mathscr{M}}$ will lead to a decomposition of $\hat{\mathscr{A}}$. Since we want to work on a separable Hilbert space we begin with the following lemma:

3.1. Lemma. Suppose $(\mathscr{A}, \mathscr{D})$ is such that $\mathscr{H}(\mathscr{D})$ is separable. If $(\hat{\mathscr{A}}, \hat{\mathscr{M}}, \hat{\mathscr{D}})$ is a regular extension, then $\mathscr{H}(\hat{\mathscr{D}})$ is also separable.

Proof. $\hat{\mathscr{D}}$ is the linear span of $\hat{\mathscr{M}} \mathscr{D}=\hat{\mathscr{M}}_{1} \mathscr{D}$, where $\hat{\mathscr{M}}_{1}$ is the unit ball in $\hat{\mathscr{M}}$. If $\mathscr{H}(\mathscr{D})$ carries the usual topology. $\mathscr{H}(\hat{\mathscr{D}})$ the weak topology and $\hat{\mathscr{M}}_{1}$ the weak operator topology, then the map $(\hat{m}, \varphi) \mapsto \hat{m} \varphi$ is separatly continuous $\hat{\mathscr{M}}_{1} \times \mathscr{H}(\mathscr{D})$ $\rightarrow \mathscr{H}(\hat{\mathscr{D}})$. The linear span of the image of this map is dense in $\mathscr{H}(\hat{\mathscr{D}})$. Since the vector addition in $\mathscr{H}(\hat{\mathscr{D}})$ is weakly continuous and weakly dense implies strongly dense for linear subspaces, it is sufficient to show that this image is separable. For this we must establish the separability of $\hat{\mathscr{M}}_{1}$. Regularity of the extension implies that $\hat{\mathscr{M}}_{1}$ can be represented as $\varrho^{-1}\left(\varrho \hat{M}_{1}\right)$ with $\varrho$ as in Definition 2.9. By Lemma 2.11 (ii) we may assume that $\hat{\mathscr{M}}$ is weakly closed, so $\hat{\mathscr{M}}_{1}$ is weakly compact. Since $\varrho$ is one-to-one when restricted to $\hat{\mathscr{M}}_{1}$ and also weakly continuous, it follows that $\varrho^{-1}$ is weakly continuous $\left([11]\right.$, p. 141). Hence, $\hat{\mathscr{M}}_{1}$ is the continuous image of $\varrho \hat{M}_{1}$, which is weakly separable because $\mathscr{H}(\mathscr{D})$ is separable.

There does not exist any general procedure for obtaining a decomposition of a family of unbounded operators. A method which fits the applications we have in mind is the use of the spectral theorem for nuclear spaces $[7,12]$. This method was first applied to the decomposition of Wightman functionals by Maurin [7, 8].

The additional hypotheses on $\mathscr{A}$ and $\mathscr{D}$ are as follows:

3.2. Assumptions. (i) $\mathscr{D}$ is a separable nuclear vector space and the imbedding $\mathscr{D} \rightarrow \mathscr{H}(\mathscr{D})$ is continuous.

(ii) $\mathscr{A}$ is a separable topological space.

(iii) The map $(A, \varphi) \mapsto A \varphi$ is separately continuous $\mathscr{A} \times \mathscr{D} \rightarrow \mathscr{D}$.

Now suppose $(\mathscr{A}, \hat{\mathscr{M}}, \hat{\mathscr{D}})$ is a maximal regular extension of $(\mathscr{A}, \mathscr{D})$. $\hat{\mathscr{M}}$ is an abelian v. Neumann algebra on a separable Hilbert space $\mathscr{H}(\mathscr{D})$ according to Lemmas 2.11 (ii) and 3.1. By the usual spectral theorem [13] there is an integral 
decomposition of $\mathscr{H}(\hat{\mathscr{D}})$ :

$$
\mathscr{H}(\hat{\mathscr{D}})=\int_{\Lambda}^{\oplus} \mathscr{H}_{\lambda} d \mu_{\lambda}
$$

with a finite positive Borel measure $d \mu$ on a locally compact space $\Lambda$ (we can even take $\Lambda$ to be the real line), such that $\hat{\mathscr{M}}$ consists of all bounded diagonal operators with respect to this decomposition. The vectors in $\mathscr{H}(\hat{\mathscr{D}})$ are represented by (equivalence classes of) measurable fields of vectors $\lambda \mapsto \varphi_{\lambda} \in \mathscr{H}_{\lambda}$. Whenever convenient we shall denote the vector corresponding to such a field by

$$
\varphi=\int^{\oplus} \varphi_{\lambda} d \mu_{\lambda}
$$

3.3. Theorem. Let $(\mathscr{A}, \mathscr{D})$ satisfy assumptions 3.2 , let $(\hat{\mathscr{A}}, \hat{\mathscr{M}}, \hat{\mathscr{D}})$ be a maximal regular extension and

$$
\mathscr{H}(\hat{\mathscr{D}})=\int_{\Lambda}^{\oplus} \mathscr{H}_{\lambda} d \mu_{\lambda}
$$

the corresponding decomposition of $\mathscr{H}(\hat{\mathscr{D}})$. $(\hat{\mathscr{A}}, \hat{\mathscr{D}})$ :

We have the following decomposition of the operator families $(\mathscr{A}, \mathscr{D})$ and

(i) For every $\lambda \in \Lambda$ there is a linear mapping $E_{\lambda}: \mathscr{D} \rightarrow \mathscr{H}_{\lambda}$ such that

(a) $\mathscr{D}_{\lambda}:=E_{\lambda} \mathscr{D}$ with the final topology is a nuclear space, continuously imbedded in $\mathscr{H}_{\lambda}$ and dense in $\mathscr{H}_{\lambda}$ for almost all $\lambda$.

(b) For all $\varphi \in \mathscr{D}, \lambda \mapsto E_{\lambda} \varphi$ is a measurable field of vectors with

$$
\varphi=\int_{\Lambda}^{\oplus} E_{\lambda} \varphi d \mu_{\lambda}
$$

(ii) For every $\lambda \in \Lambda$ there is a mapping $A \rightarrow A_{\lambda}$ of $\mathscr{A}$ into linear operators on $\mathscr{D}_{\lambda}$ such that

(a) For almost all $\lambda$ we have $E_{\lambda}(A \varphi)=A_{\lambda} E_{\lambda} \varphi$ for all $A \in \mathscr{A}$ and $\varphi \in \mathscr{D}$, and therefore

for $A \in \mathscr{A}, \varphi \in \mathscr{D}$.

$$
A \varphi=\int_{\Lambda}^{\oplus} A_{\lambda} E_{\lambda} \varphi d \mu_{\lambda}
$$

(b) The mapping $A \rightarrow A_{\lambda}$ preserves all algebraic relations and the involution.

(c) If we equip the set $\mathscr{A}_{\lambda}$ of all $A_{\lambda}$ with the final topology relative to the map $A \rightarrow A_{\lambda}$, then those of the operations in (b) which are continuous on $\mathscr{A}$ remain continuous on $\mathscr{A}_{\lambda}$.

(d) $\left(A_{\lambda}, \varphi_{\lambda}\right) \mapsto A_{\lambda} \varphi_{\lambda}$ is a separately continuous map $\mathscr{A}_{\lambda} \times \mathscr{D}_{\lambda} \rightarrow \mathscr{D}_{\lambda}$.

(iii) If $\varphi$ is any vector in $\hat{\mathscr{D}}$ and $\lambda \mapsto \varphi_{\lambda} \in \mathscr{H}_{\lambda}$ any measurable field representing $\varphi$, then

(a) $\varphi_{\lambda} \in \mathscr{D}_{\lambda}$ a.e.

(b) $\lambda \mapsto A_{\lambda} \varphi_{\lambda}$ is measurable for all $A \in \mathscr{A}$, and

$$
\hat{A} \varphi=\int_{\Lambda}^{\oplus} A_{\lambda} \varphi_{\lambda} d \mu_{\lambda}
$$

where $\hat{A}$ denotes the extension of $A$ to $\hat{\mathscr{D}}$. 
(iv) If $(\mathscr{A}, \mathscr{D})$ is cyclic, i.e. $\mathscr{D}=\mathscr{A} \Omega$ with some vector $\Omega \in \mathscr{D}$, then also $\mathscr{D}{ }_{\lambda}=\mathscr{A}_{\lambda} \Omega_{\lambda}$ a.e. with $\Omega_{\lambda}=E_{\lambda} \Omega \in \mathscr{D}_{\lambda}$.

(v) $\left(\mathscr{A}_{\lambda}, \mathscr{D}_{\lambda}\right)_{w}^{\prime}$ contains only multiples of the identity operator on $\mathscr{H}_{\lambda}$ a.e.

Proof. (i) The existence of a continuous linear mapping $E_{\lambda}: \mathscr{D} \rightarrow \mathscr{H}_{\lambda}$ such that $\varphi=\int^{\oplus} E_{\lambda} \varphi d \mu_{\lambda}$ is guaranteed by the nuclear spectral theorem (see [12], Chapter I, $\S 4,[7]$, Chapter II, $\S 3$ ) (it is immaterial whether $\mathscr{D}$ is dense in $\mathscr{H}(\hat{D})$ or not). Since $E_{\lambda}$ is continuous, its kernel is closed, and $\mathscr{D}_{\lambda} \cong \mathscr{D} / \operatorname{Ker} E_{\lambda}$ therefore nuclear. That $\mathscr{D}_{\lambda}$ is dense in $\mathscr{H}_{\lambda}$ will follow from (iii) (a).

(ii) Let us consider the expression

$$
T_{\lambda}(\varphi, \psi, A)=\left\langle E_{\lambda} \varphi, E_{\lambda} A \psi\right\rangle-\left\langle E_{\lambda} A^{*} \varphi, E_{\lambda} \psi\right\rangle
$$

for $\varphi, \psi \in \mathscr{D}, A \in \mathscr{A}$. We claim that $T_{\lambda} \equiv 0$ a.e. $T_{\lambda}$ is separately continuous in its variables, so it is sufficient to check this for dense subsets of $\mathscr{D}$ and $\mathscr{A}$. Both spaces are separable by assumption and since a countable union of null sets is a null set we can restrict ourselves to one fixed triple $(\varphi, \psi, A)$. Let $m(\lambda)$ be an arbitrary bounded measurable function on $\Lambda$, and $\hat{m}$ the corresponding operator in $\hat{\mathscr{M}}$. We then have, because $\hat{m} \in(\hat{\mathscr{A}}, \hat{\mathscr{D}})_{s}^{\prime}$ :

$$
\int m(\lambda) T_{\lambda}(\varphi, \psi, A) d \mu_{\lambda}=\langle\varphi, \hat{m} A \psi\rangle-\left\langle A^{*} \varphi, \hat{m} \psi\right\rangle=0 .
$$

Since $m(\lambda)$ was arbitrary it follows that $T_{\lambda}$ vanishes for almost all $\lambda$. Consider any such $\lambda$ and let $E_{\lambda} \varphi$ with $\varphi \in \mathscr{D}$ be an element of $\mathscr{D}_{\lambda}$ and $A \in \mathscr{A}$. We define

$$
A_{\lambda}\left(E_{\lambda} \varphi\right):=E_{\lambda}(A \varphi) \text {. }
$$

This is well defined, because if $T_{\lambda} \equiv 0$, then

$$
\left\langle E_{\lambda} A \varphi, E_{\lambda} A \varphi\right\rangle=\left\langle E_{\lambda} \varphi, E_{\lambda} A^{*} A \varphi\right\rangle
$$

so $E_{\lambda} \varphi=0$ implies $E_{\lambda} A \varphi=0$. For $\lambda$ in the null set with $T_{\lambda} \neq 0$ we define $A_{\lambda}=0$. From the definition of $A_{\lambda}$ it is straightforward to verify that $\left(A_{\lambda}\right)^{*}=A_{\lambda}^{*},(A+\alpha B)_{\lambda}$ $=A_{\lambda}+\alpha B_{\lambda}$ and $(A \cdot B)_{\lambda}=A_{\lambda} \cdot B_{\lambda}$. If $A_{\lambda}=0$, then $A_{\lambda}$ is trivially continuous. For those $\lambda$ with $T_{\lambda}=0$ we have that $A$ leaves $\operatorname{ker} E_{\lambda}$ invariant. Since $A$ is continuous on $\mathscr{D}$ it follows that $A_{\lambda}$ is continuous on $\mathscr{D}_{\lambda} \cong \mathscr{D} / \operatorname{ker} E_{\lambda}$. The assertions (c) and (d) are obvious.

(iii) The vectors $\varphi \in \hat{\mathscr{D}}$ have the form $\sum_{i=1}^{n} \hat{m}_{i} \psi_{i}$ with $m_{i} \in \hat{\mathscr{M}}$ and $\psi_{i} \in \mathscr{D}$. It is sufficient to consider vectors of the form $\varphi=\hat{m} \psi$. Every $\hat{m} \in \hat{\mathscr{M}}$ is given by a measurable field $\lambda \mapsto m(\lambda) 1_{\lambda}$, where $m(\lambda) \in \mathbb{C}$ and $1_{\lambda}$ is the unit operator on $\mathscr{H}_{\lambda}$. Since $\psi=\int^{\oplus} E_{\lambda} \psi d \mu_{\lambda}$, we have that $\varphi=\hat{m} \psi$ is represented by the field $\lambda \mapsto m(\lambda)$ - $E_{\lambda} \varphi \in \mathscr{D}_{\lambda}$ which proves (a). To prove (b) we have only to note that $\hat{A} \varphi=\hat{m} A \psi$ so

$$
\begin{aligned}
\hat{A} \varphi & =\int^{\oplus} m(\lambda) A_{\lambda} E_{\lambda} \varphi d \mu_{\lambda}=\int^{\oplus} A_{\lambda}\left(m(\lambda) E_{\lambda} \psi\right) d \mu_{\lambda} \\
& =\int^{\oplus} A_{\lambda} \varphi_{\lambda} d \mu_{\lambda} \quad \text { because } \varphi=\int^{\oplus} m(\lambda) E_{\lambda} \psi d \mu_{\lambda} .
\end{aligned}
$$

Since $\hat{\mathscr{D}}$ is dense in $\mathscr{H}(\hat{\mathscr{D}})$ it follows from (a) that $\mathscr{D}_{\lambda}$ is dense in $\mathscr{H}_{\lambda}$ a.e.

(iv) Follows from (ii) (a). 
For the proof of the last statement (v) we need a result on the decomposability of the weak commutant:

3.4. Lemma. Under the assumptions of Theorem 3.3 there exist measurable fields of bounded operators $\lambda \mapsto c_{i}(\lambda), i=1,2, \ldots$, such that $\left\{c_{i}(\lambda)\right\}_{i=1,2 \ldots}$ is weakly dense in $\left(\mathscr{A}_{\lambda}, \mathscr{D}_{\lambda}\right)_{w}^{\prime}$ for almost every $\lambda$.

Proof. The proof is an adaption of the corresponding proof for the commutant of a measurable field of v. Neumann algebras, cf. Dixmier [13], Lemme 1, p. 183. Since $\mathscr{H}(\hat{\mathscr{D}})$ is separable by Lemma 3.1 , the measure appearing in the decomposition of $\hat{\mathscr{M}}$ is a regular Borel measure on a locally compact space with a countable base. (In fact we can take this space to be the real line.) As in [13], it is sufficient to consider the case where $\mathscr{H}_{\lambda}=H$ is independent of $\lambda$. Let $\mathscr{B}(H)$ denote the bounded operators on $H$ and $\mathscr{B}_{1}(H)$ the unit ball in $\mathscr{B}(H)$. An operator $c \in \mathscr{B}(H)$ is in $\left(\mathscr{A}_{\lambda}, \mathscr{D}_{\lambda}\right)_{w}^{\prime}$ if and only if

$$
\left\langle E_{\lambda} \varphi, c E_{\lambda} A \psi\right\rangle-\left\langle E_{\lambda} A^{*} \varphi, c E_{\lambda} \psi\right\rangle=0
$$

for all $\varphi, \psi \in \mathscr{D} . A \in \mathscr{A}$. For fixed $\lambda, c$ this expression is separately continuous in the variables $\varphi, \psi, A$, so it vanishes everywhere if it vanishes on a dense set. Let $\left\{\varphi_{j}\right\}$ and $\left\{A_{k}\right\}$ be countable dense sets in $\mathscr{D}$ and $\mathscr{A}$ respectively and denote by $T_{i}(\lambda, c)$ the countably many functions of $c$ and $\lambda$ of the form above, where $\varphi, \psi$, and $A$ run through these sets. Thus, $c \in\left(\mathscr{A}_{\lambda}, \mathscr{D}_{\lambda}\right)_{w}^{\prime}$ if and only if $T_{i}(\lambda, c)=0$ for all $i=1,2, \ldots T_{i}(\lambda, c)$ is weakly continuous in $c$ for fixed $\lambda$. Moreover, we can by Lusin's theorem write our measure space, up to a null set, as a countable union of compact sets $Y_{k}$ such that the mappings $\lambda \mapsto E_{\lambda} \varphi_{i}$ and $\lambda \mapsto E_{\lambda} A_{j} \varphi_{l}$ are all continuous mappings from $Y_{k} \rightarrow H$. It is sufficient to consider one such compact set $Y$, and $T_{i}$ is easily seen to be jointly continuous on $Y \times \mathscr{B}_{1}(H)$ because

$$
\begin{aligned}
\mid\left\langle\varphi_{\lambda_{0}}, c_{0}\right. & \left.\psi_{\lambda_{0}}\right\rangle-\left\langle\varphi_{\lambda}, c \psi_{\lambda}\right\rangle \mid \\
\leqq & \left|\left\langle\varphi_{\lambda_{0}},\left(c_{0}-c\right) \psi_{\lambda_{0}}\right\rangle\right|+\left|\left\langle\varphi_{\lambda_{0}}-\varphi_{\lambda}, c \psi_{\lambda_{0}}\right\rangle\right| \\
& \quad+\left|\left\langle\left(\varphi_{\lambda_{0}}-\varphi_{\lambda}\right), c\left(\psi_{\lambda_{0}}-\psi_{\lambda}\right)\right\rangle\right|+\left|\left\langle\varphi_{\lambda_{0}}, c\left(\psi_{\lambda_{0}}-\psi_{\lambda}\right)\right\rangle\right|
\end{aligned}
$$

As a consequence, $\mathfrak{M}=\bigcap_{i} \operatorname{ker} T_{i}$ is a closed subset of $Y \times \mathscr{B}_{1}(H)$ which is compact and metrizable with a countable base.

The remaining part of the proof goes exactly as in [13], Lemme 1, pp. 183-184, and will not be repeated here.

With this result we can now prove the remaining statement (v) of Theorem 3.3:

Let $S$ denote the set of all $\lambda$ such that $\left(\mathscr{A}_{\lambda}, \mathscr{D}_{\lambda}\right)_{w}^{\prime}$ is trivial and $S_{i}$ the set of all $\lambda$ such that $c_{i}(\lambda)$ in Lemma 3.4 is trivial. $S_{i}$ is a measurable set, because $\lambda \mapsto c_{i}(\lambda)$ is measurable and the multiples of the identity are closed in $\mathscr{B}(H)$. By Lemma 3.4, $S=\bigcap_{i} S_{i}$. If the complement of $S$ is not a null set, then this must also be the case for at least one $S_{i}$. The operator $c_{i}$ corresponding to the measurable field $\lambda \mapsto c_{i}(\lambda)$ is then not in $\hat{\mathscr{M}}$ although it is clearly in $(\hat{\mathscr{A}}, \hat{\mathscr{M}}, \hat{\mathscr{D}})_{w}^{\prime}$. This contradicts the maximality of the extension $(\hat{\mathscr{A}}, \hat{\mathscr{M}}, \hat{\mathscr{D}})$ by Theorem 2.16 .

3.5. Remark. We point out that we did not need any nuclear topology on $\hat{\mathscr{D}}$ for the proof of Theorem 3.3. The reason was simply that $\hat{\mathscr{D}}$ is equal to the linear span of $\hat{\mathscr{M}} \mathscr{D}$, and $\hat{\mathscr{M}}$ is diagonalized by the decompositon, so the spaces $\mathscr{D}_{\lambda}$ associated 
with $\mathscr{D}$ give automatically a decomposition of $\hat{\mathscr{D}}$. It might however be of some interest that one can always extend the nuclear topology of $\mathscr{D}$ to a subset of $\hat{\mathscr{D}}$ which is dense in the graph topology induced by $\hat{\mathscr{A}}$.

3.6. Lemma. Suppose $(\mathscr{A}, \mathscr{D})$ satisfies the assumption 3.2 and let $(\hat{\mathscr{A}}, \hat{\mathscr{M}}, \hat{\mathscr{D}})$ be a regular induced extension with $\mathscr{M}$ norm closed. Then there is a strongly dense nuclear sub *-algebra $\hat{\mathscr{N}} \subset \hat{\mathscr{M}}$ such that 3.2 .

(i) $(\tilde{\mathscr{A}}, \tilde{\mathscr{N}}, \tilde{\mathscr{D}})$ is a regular induced extension, and $(\tilde{\mathscr{A}}, \tilde{\mathscr{D}})$ satisfies the assumptions

(ii) The extensions $(\tilde{\mathscr{A}}, \tilde{\mathscr{D}})$ and $(\hat{\mathscr{A}}, \hat{\mathscr{D}})$ have the same closure.

Proof. By the spectral theorem and because $\mathscr{H}(\hat{\mathscr{D}})$ is separable, $\hat{\mathscr{M}}$ contains a strongly dense subalgebra which is isomorphic to the quotient algebra of the bounded continuous functions on the real line modulo the closed ideal of functions vanishing a.e. with respect to some regular Borel measure. The bounded continuous functions on $\mathbb{R}$ contain as a strongly dense nuclear subalgebra the Schwartz space $\mathscr{S}$ of rapidly decreasing $C^{\infty}$-functions. We define $\tilde{\mathscr{N}}$ as the corresponding nuclear quotient algebra which is then dense in $\hat{\mathscr{M}}$.

$\tilde{\mathscr{D}}=$ linear span $\tilde{\tilde{N}} \mathscr{D}$ is as a vector space isomorphic to the topological tensor product $\tilde{\mathscr{N}} \otimes_{\pi} \mathscr{D}$ modulo a closed subspace and we define the topology on $\tilde{\mathscr{D}}$ to be this quotient topology. Since $\tilde{\mathscr{A}}$, the extension of $\mathscr{A}$ to $\tilde{\mathscr{D}}$, is a faithful representation of $\mathscr{A}$ we can simply define the topology on $\mathscr{A}$ to be the same as on $\mathscr{A}$. It is straightforward to verify condition (iii) of 3.2. Finally, $\tilde{\mathscr{D}}$ is dense in the graph topology of $\hat{\mathscr{D}}=$ linear span $\hat{\mathscr{M}} \mathscr{D}$, because $\tilde{\mathscr{N}}$ is strongly dense in $\hat{\mathscr{M}}$ and $\|\hat{A} \hat{m} \varphi\|=\|\hat{m} \hat{A} \varphi\|$, so if $\hat{m}_{\alpha} \in \tilde{\mathscr{N}}$ converges strongly to $\hat{m} \in \hat{\mathscr{M}}$, then $\hat{m}_{\alpha} \varphi \in \tilde{\mathscr{D}}$ converges to $\hat{m} \varphi$ in the graph norm of every operator $\hat{A} \in \hat{\mathscr{A}}$.

We now want to apply the results obtained so far to representations of nuclear algebras and the problem of decomposing a positive functional into extreme states. This needs a little preparation because in this case we have usually only given a topology on the operators and must first define a topology on the domain $\mathscr{D}$ such that the assumptions 3.2 are satisfied. We shall use the following terminology: For topological algebras we require the product of two elements only to be separately continuous. If the algebra has a unit $I$, we call a positive linear functional $\omega$ a state if $\omega(I)=1$. By "nuclear $*$-algebra" we mean a topological $*$-algebra with a locally convex nuclear topology. We call a representation $\pi$ of a topological algebra $\mathfrak{A}$ with domain $\mathscr{D}_{\pi}$ in a Hilbert space $\mathscr{H}_{\pi}$ weakly continuous if $\langle\varphi, \pi(A) \psi\rangle$ is continuous in $A$ for all $\varphi, \psi \in \mathscr{D}_{\pi}$, and strongly continuous if $A \mapsto \pi(A) \varphi$ is a continuous mapping $\mathfrak{U} \rightarrow \mathscr{H}_{\pi}$ for all $\varphi \in \mathscr{D}_{\pi}$. If the multiplication is jointly continuous, the latter is implied by the former for a $*$-algebra, and according to the following simple criterion, (cf. [14], Theorem 4.1), this is also true in most other cases of interest:

3.7. Lemma. For a barrelled topological algebra every weakly continuous representation is strongly continuous.

Proof. For every $\varphi \in \mathscr{D}_{\pi}$ and $A \in \mathfrak{A}$ we have

$$
\|\pi(A) \varphi\|=\sup _{\substack{\psi \in \mathscr{D} \pi \\\|\psi\|=1}}|\langle\psi, \pi(A) \varphi\rangle| .
$$


Since $\pi$ is weakly continuous, the set

$$
\{A \in \mathfrak{A}||\langle\psi, \pi(A) \varphi\rangle \mid \leqq 1\}
$$

is closed for all $\varphi, \psi \in \mathscr{D}$. The same holds for the absolutely convex and absorbing set

$$
\{A \mid\|\pi(A) \varphi\| \leqq 1\}=\bigcap_{\substack{\psi \in \mathscr{D} \pi \\\|\psi\|=1}}\{A||\langle\psi, \pi(A) \varphi\rangle \mid \leqq 1\}
$$

which is thus a neighbourhood of zero since $\mathfrak{A}$ is barrelled.

Given a continuous representation of a nuclear *-algebra, we can use the topology of the algebra to define a nuclear topology at least on an invariant subspace of the domain of definition:

3.8. Lemma. Let $\pi$ be a strongly continuous representation of a nuclear $*$-algebra $\mathfrak{U}$ on a dense linear domain $\mathscr{D}$ in a separable Hilbert space $\mathscr{H}(\mathscr{D})$. Then there is a dense linear domain $\mathscr{D}_{0} \subset \mathscr{D}$ and a nuclear topology on $\mathscr{D}_{0}$ such that

(i) $\mathscr{D}_{0}$ is invariant under the representation, and every $\pi(A)$ is a continuous operator $\mathscr{D}_{0} \rightarrow \mathscr{D}_{0}$.

(ii) The imbedding $\mathscr{D}_{0} \rightarrow \mathscr{H}(\mathscr{D})$ is continuous.

(iii) $\mathscr{D}_{0}$ is separable if $\mathfrak{A}$ is separable.

Moreover, if $\left\{\Omega_{i}\right\}$ is any countable subset of $\mathscr{D}$ there is a $\mathscr{D}_{0}$ as above with $\left\{\Omega_{i}\right\} \subset \mathscr{D}_{0}$.

Proof. The statement is a simple consequence of the fact that nuclearity is preserved under the operations of taking quotients with respect to closed subspaces and forming countable direct sums. Let $\left\{\Omega_{i}\right\}$ be any countable subset of $\mathscr{D}$. If $\mathscr{D}_{0}:=$ linear span $\left\{\Omega_{i}\right\} \cup\left\{\pi(\mathfrak{U}) \Omega_{i}\right\}$ is not already dense in $\mathscr{D}$, we add countably many elements to the set $\left\{\Omega_{i}\right\}$ until it becomes dense and then define $\mathscr{D}_{0}$ in the same way with this extended set. For every $i$ we define a nuclear topology on the space $\pi(\mathfrak{U}) \Omega_{i}$ as the quotient topology of $\mathfrak{A}$ modulo the kernel of the continuous map $A \mapsto \pi(A) \Omega_{i}$. The one-dimensional space generated by $\Omega_{i}$ is trivially nuclear. $\mathscr{D}_{0}$ is as a vector space isomorphic to the direct sum of all these countably many spaces modulo a closed subspace and we define the topology of $\mathscr{D}_{0}$ accordingly. The other properties of $\mathscr{D}_{0}$ are readily verified.

3.9. Remarks. (a) If the representation is cyclic, i.e. $\mathscr{D}=\pi(\mathfrak{U}) \Omega$ with some $\Omega \in \mathscr{D}$, then we can of course take $\mathscr{D}_{0}=\mathscr{D}$. If this should not be possible, it might sometimes be desirable that $\mathscr{D}_{0}$ is at least dense in the graph-topology so that $\left(\pi(\mathfrak{U}), \mathscr{D}_{0}\right)$ and $(\pi(\mathfrak{A}), \mathscr{D})$ have the same closure. This can obviously be achieved if $\mathscr{D}$ is separable in the graph-topology. We do not want to pursue the question here when this is the case, but only remark that it is certainly sufficient that $\pi(\mathfrak{A})$ is dominated by a countable subset, i.e. there are countably many $A_{i} \in \mathfrak{A}$, such that for every $A \in \mathfrak{U}$ there is an $A_{i}$ with $\|\pi(A) \varphi\| \leqq\left\|\pi\left(A_{i}\right) \varphi\right\|$ for all $\varphi \in \mathscr{D}$.

(b) In Lemma 3.8 the topology on $\mathfrak{A}$ was given and the problem was to define a suitable topology on the domain $\mathscr{D}$. In the opposite situation when $\mathscr{D}$ is a given nuclear space we can for the continuous operators on $\mathscr{D}$ use the topology of uniform convergence on bounded sets. If the strong dual $\mathscr{D}^{\prime}$ is nuclear, this is a nuclear topology [15], because the continuous operators on $\mathscr{D}$ can be identified with a subspace of the complete tensor product $\mathscr{D} \hat{\otimes} \mathscr{D}^{\prime}$. If $\mathscr{D}$ and $\mathscr{D}^{\prime}$ are separable, this space is also separable. 
As a corollary to Theorems 3.3 and 2.16 we can now state

3.10. Theorem. (i) Suppose $\pi$ is a strongly continuous representation of a separable, nuclear *-algebra $\mathfrak{A}$ on a dense set $\mathscr{D}$ in a separable Hilbert space $\mathscr{H}$. Then there is a separable Hilbert space $\hat{\mathscr{H}}$, containing $\mathscr{H}$ as a closed subspace, a direct integral decomposition

$$
\hat{\mathscr{H}}=\int^{\oplus} \mathscr{H}_{\lambda} d \mu_{\lambda}
$$

where $d \mu$ is a finite positive Borel measure on the real line, and strongly continuous representations $\pi_{\lambda}$ of $\mathfrak{U}$ on dense subspaces $\mathscr{D}_{\lambda} \subset \mathscr{H}_{\lambda}$ such that

(a) For every $\varphi$ in a dense set $\mathscr{D}_{0} \subset \mathscr{D}$ and any measurable field $\lambda \mapsto \varphi_{\lambda}$ with $\varphi=\int^{\oplus} \varphi_{\lambda} d \mu_{\lambda}$ we have $\varphi_{\lambda} \in \mathscr{D}_{\lambda}$ a.e. and

$$
\pi(A) \varphi=\int^{\oplus} \pi_{\lambda}(A) \varphi_{\lambda} d \mu_{\lambda} .
$$

(b) $\operatorname{ker} \pi \subset \operatorname{ker} \pi_{\lambda}$ a.e.

(c) $\left(\pi_{\lambda}(\mathfrak{U}), \mathscr{D}_{\lambda}\right)_{w}^{\prime}=\left\{c \cdot 1_{\lambda} \mid c \in \mathbb{C}\right\}$ a.e.

(ii) Let $\mathfrak{A}$ be a nuclear and separable *-algebra with a unit element. Suppose $\mathfrak{U}$ is either barrelled, or the multiplication on $\mathfrak{U}$ is jointly continuous. Then every positive, continuous linear functional $\omega$ on $\mathfrak{A}$ has a decomposition

$$
\omega=\int \omega_{\lambda} d \mu_{\lambda},
$$

where $d \mu$ is a measure as in (i) and $\omega_{\lambda}$ is an extremal state for almost all $\lambda$. The integral is to be understood in the weak sense.

For the left kernels $L\left(\omega_{\lambda}\right)=\left\{A \mid \omega_{\lambda}\left(A^{*} A\right)=0\right\}$ we have

$$
L(\omega) \subset L\left(\omega_{\lambda}\right) \text { a.e. }
$$

Proof. (i) If we choose $\mathscr{D}_{0}$ as in Lemma 3.8 and put the quotient topology of $\mathfrak{U} / \operatorname{ker} \pi$ on $\pi(\mathfrak{U})$, then $\left(\pi(\mathfrak{U}), \mathscr{D}_{0}\right)$ satisfies the hypotheses of Theorem 3.3. The existence of a maximal regular extension is guaranteed by Theorem 2.16. The statements (a) and (c) are thus immediate consequences of the previous results. As to (b) we note that $\pi(A)=0$ implies $\pi_{\lambda}(A)=0$ for all $\lambda$ outside a null set which might depend on $A$. To get a common null set we appeal to separability of $\mathfrak{U}$ and continuity of the representation.

(ii) A continuous positive functional defines a weakly continuous cyclic representation $\pi$ of $\mathfrak{U}$ with cyclic vector $\Omega$ and $\mathscr{D}=\pi(\mathfrak{A}) \Omega$. If $\mathfrak{A}$ is barrelled, this representation is strongly continuous according to Lemma 3.7. Obviously, this holds also if the multiplication is jointly continuous. By Lemma 3.8 we can define a nuclear topology on $\mathscr{D}$ and then apply Theorem 3.3. The representations $\pi_{\lambda}$ appearing in the decomposition are also cyclic and we have $\omega(A)=\int \omega_{\lambda}(A) d \mu_{\lambda}$ with $\omega_{\lambda}(A)=\left\langle\Omega_{\lambda}, \pi_{\lambda}(A) \Omega_{\lambda}\right\rangle$. The $\omega_{\lambda}$ are positive functionals, so

$$
0=\omega\left(A^{*} A\right)=\int \omega_{\lambda}\left(A^{*} A\right) d \mu_{\lambda}
$$

implies $\omega_{\lambda}\left(A^{*} A\right)=0$ a.e. By separability of $\mathfrak{A}$ and continuity of $\omega_{\lambda}$ we get a null set independent of $A$ so

$$
L(\omega) \subset L\left(\omega_{\lambda}\right) \text { a.e. }
$$


Since $\mathfrak{A}$ has a unit $I$ we can normalize $\omega_{\lambda}$ by dividing with $\omega_{\lambda}(I)^{-1}$, because $\omega_{\lambda}(I)=0$ implies $\omega_{\lambda}=0$.

Finally it follows from (i) (c) that $\omega_{\lambda}$ is an extremal state for almost all $\lambda$, because a positive functional is extremal if and only if the corresponding representation has a trivial weak commutant, cf. e.g. Powers [10], Theorem 6.3.

\section{Applications to Wightman Theory}

The results of the previous section apply in particular to the tensor algebra $\mathscr{\mathscr { S }}$ over Schwartz space [1-3], or any other space of test functions, provided it is nuclear, separable and barrelled. We recall that a state on $\mathscr{\mathscr { S }}$ is called a Wightman state if it is invariant under the Poincaré group and vanishes on two prescribed ideals $\mathscr{I}_{\mathrm{Sp}}$ and $\mathscr{I}_{c}[1-3]$. The representation of $\mathscr{S}$ corresponding to such a functional defines a Wightman field satisfying the conditions of local commutativity, relativistic invariance, positive energy and existence of a cyclic invariant state.

From Theorem 3.10 (ii) we have the following result:

4.1. Theorem. Every state $W$ on $\mathscr{\mathscr { S }}$ has a weak integral decomposition

$$
W=\int W_{\lambda} d \mu_{\lambda}
$$

with a positive Borel measure $d \mu_{\lambda}$ on the real line, and $W_{\lambda}$ extreme for almost all $\lambda$.

If $W$ is a Wightman state, this is also the case for $W_{\lambda}$ a.e.

Proof. The first statement follows immediately from Theorem 3.10 (ii), because $\mathscr{S}$ is a nuclear, separable and barrelled $*$-algebra $[2,3]$.

If $W$ is a Wightman state, then the ideals $\mathscr{I}_{\mathrm{Sp}}$ and $\mathscr{I}_{c}$ are contained in the left kernel $L(W)$. Since $L(W) \subset L\left(W_{\lambda}\right)$, the components of $W$ will also satisfy locality and spectrum condition. As to the invariance of $W_{\lambda}$, we note the result of [1] that every bounded operator which commutes weakly with the field commutes also with the representation of the Poincaré group. Hence, if $\hat{\mathscr{M}}$ is the abelian algebra defining the decomposition of the extended Hilbert space $\hat{\mathscr{H}}$ then $\hat{\mathscr{M}}$ commutes weakly with the group representation on the original Hilbert space $\mathscr{H}$. The reader will easily convince himself that this is sufficient for the invariance of $W_{\lambda}$. Alternatively, one can extend the representation from $\mathscr{H}$ to $\hat{\mathscr{H}}$ by defining $\hat{U}(a, \Lambda) \hat{m} \varphi=\hat{m} U(a, \Lambda) \varphi$. One shows that this is well defined and $\hat{U}$ commutes strongly with $\hat{\mathscr{M}}$ on $\hat{\mathscr{H}}$.

Although we have stated this theorem for the special case of a cyclic representation of $\mathscr{L}$, the decomposition theory of the preceding section can be used in more general situations if needed. (E.g. if one is interested in the decomposition of a subalgebra of the algebra of field operators.) The only problem is to choose a suitable nuclear domain of definition for the field operators and the group. If there is no natural domain at hand we can always construct a domain as in Lemma 3.8. This domain will be invariant under the Poincaré group if the vectors $\Omega_{i}$ are invariant. If this should not be the case one can use the fact that the Poincare group is a Lie group and smear the operators of the group representations with $C^{\infty}$-functions with compact support on the group manifold. These functions define a nuclear topology on the smeared operators, so one can again use Lemma 3.8, 
where $\mathscr{A}$ is now the algebra generated by the field operators and the smeared group operators. This method for obtaining an invariant nuclear domain can of course also be used for any other symmetry group which might be present, provided it is a Lie group. Because of Theorem 3.10 (i) (b), a local field will decompose into local fields. We already mentioned the fact [1] that a decomposition of the field will automatically lead to a decomposition of the representation of the Poincaré group, at least if there is a cyclic set of invariant vectors and the field is tempered. Otherwise one has to require separately that the operators defining the decomposition commute also with the group. Extending the group representation to the extended Hilbert space $\hat{\mathscr{H}}$ does not cause any difficulties and a representation satisfying the spectrum condition will also decompose into such representations.

Since we have now obtained a decomposition of every Wightman state into extreme states, an important and natural question which arises is the following: Do extreme Wightman states necessarily have the cluster property, i.e. is the vacuum unique for a theory corresponding to an extreme state? The converse has been known to be true for a long time [16], and within the framework of local algebras of bounded operators, both conditions are equivalent [17]. In [18] it was shown that due to the pathologies associated with unbounded operators it is possible for a field to have a trivial strong commutant and still have a degenerate vacuum. We now want to show by an analogous example that this can even happen for a field with a trivial weak commutant, so uniqueness of the vacuum is not equivalent to the state being extreme.

4.2. Example. In [10], Powers gave an example of a representation of a commutative algebra on an infinite demensional Hilbert space such that the weak commutant of the representation is trivial. Incidentally, the existence of such a representation can also be shown as follows: According to an example of Hilbert (see e.g. [12]) a polynomial in two or more variables can be positive for all real values of its variables without being the sum of squares of polynomials. With the aid of the Hahn-Banach theorem one concludes from this that there exists a linear functional $T$ on the free abelian algebra of $n$ generators, $n \geqq 2$, such that $T$ is positive, but not strongly positive [12]. This algebra is the symmetric tensor algebra over the nuclear space $\mathbb{C}^{n}$, so we can apply the decomposition theory of Section 3 and obtain an extremal state which is not strongly positive. Because a positive function of bounded commuting Hermitean operators is positive, the corresponding representation must be unbounded and therefore infinite dimensional.

Suppose now that $G_{1}, \ldots, G_{n}$ are the Hermitean generators of such a representation with cyclic vector $\Omega_{0}$ and domain $\mathscr{D}_{0}$ in an infinite dimensional Hilbert space $\mathscr{H}_{0}$. Define $B_{i}=1+G_{i}$ for $i=1, \ldots, n$ and $B_{i}=1-G_{i}$ for $i=n+1, \ldots, 2 n$. Let $A_{i}(x)$ be $2 n$ commuting free fields to different masses $m_{i}$, with a cyclic vacuum $\Omega_{1}$ and domain $\mathscr{D}_{1}$ in a Hilbert space $\mathscr{H}_{1}$. We can take $\mathscr{H}_{1}$ as the tensor product of the Fock spaces associated with the different fields and $\Omega_{1}$ as the tensor product of the vacua in these spaces. We now define a field $A(x)$ on $\mathscr{D}_{0} \otimes \mathscr{D}_{1} \subset \mathscr{H}_{0} \otimes \mathscr{H}_{1}$ :

$$
A(x)=\sum_{i=1}^{2 n} B_{i} \otimes A_{i}(x)
$$


and a representation of the Poincaré group

$$
U(a, \Lambda)=1 \otimes U_{1}(a, \Lambda),
$$

where $U_{1}(a, \Lambda)$ is the representation of the group on $\mathscr{H}_{1}$. The invariant vectors span the infinite dimensional subspace $\mathscr{H}_{0} \otimes \Omega_{1}$. If we smear $A(x)$ with test functions which are concentrated in momentum space around the mass shell $m_{i}$, the contributions from the $A_{j}(x)$ with $j \neq i$ vanish, so the algebra generated by all $A(f)$ 's is the same as the algebra generated by all operators $B_{1} \otimes A_{1}(f), \ldots$, $B_{2 n} \otimes A_{2 n}(f)$. Although $\Omega:=\Omega_{0} \otimes \Omega_{1}$ need not be a cyclic vector w.r.t. the whole Hilbert space $\mathscr{H}_{0} \otimes \mathscr{H}_{1}$, it is easy to verify that the subspace $\mathscr{H}$, generated by applying polynomials in the field to $\Omega$, contains all the invariant vectors $\mathscr{H}_{0} \otimes \Omega_{1}$. Indeed, if $f$ has support in momentum space around the negative mass shell $m_{i}$, then $A_{i}\left(f^{*}\right) A_{i}(f) \Omega_{1}$ is a multiple of $\Omega_{1}$. Moreover the algebra generated by the $B_{i}^{2}$ 's is the same as the algebra generated by the $G_{i}$ 's, so repeated application of $B_{i}^{2} \otimes A_{i}(f)^{*} A_{i}(f)$ to $\Omega_{0} \otimes \Omega_{1}$ gives $\mathscr{H}_{0} \otimes \Omega_{1}$. Suppose $C$ is a bounded operator on $\mathscr{H}$ commuting weakly with the field. By Theorem 4 in [1] $C$ commutes with the representation of the Poincaré-group and therefore with the projector $P_{0}$ on the invariant states. The restriction of $C$ to the subspace of invariant states commutes with all the operators $B_{i}^{2} \otimes A_{i}\left(f^{*}\right) A_{i}(f)$, which leave this space invariant if $f$ has support around the negative mass shell $m_{i}$. By irreducibility of the $B_{i}^{2}$,s, this restriction of $C$ is a multiple of the identity operator. Since the invariant states are cyclic for $A(f)$ in $\mathscr{H}$ they are separating for the weak commutant, so $C$ itself is a scalar operator.

As indicated by this example and the considerations in [18], the problem of finding conditions on the field under which it can be decomposed into fields with only one vacuum amounts to finding conditions for the solvability of a moment problem for an abelian algebra, namely the algebra generated by operators of the form $P_{0} A(\underline{f}) P_{0}$, where $A(\underline{f})$ is a polynomial in smeared field operators and $P_{0}$ the projector on the invariant states. It was shown in [18], that these operators are well defined and commute with each other. In particular, it follows from Theorem 3 in [18], that a field with a trivial strong commutant has either a unique vacuum or an infinitely degenerate vacuum. We shall discuss these questions more thoroughly in a subsequent paper.

\section{References}

1. Borchers, H. J.: Nuovo Cimento 24, 1118-1140 (1962)

2. Borchers, H.J.: Algebraic aspects of Wightman field theory. In: Sen, R.N., Weil,C. (Eds.): Statistical mechanics and field theory. Lectures given at the 1971 Haifa Summer School. New York: Halsted Press 1972

3. Wyss, W.: On Wightman's theory of quantized fields. Lectures in theoretical physics. University of Colorado, Boulder 1968. New York: Gordon and Breach 1969

4. Choquet, G.: Lectures on analysis. New York: Benjamin 1969

5. Wyss, W.: Commun. math. Phys. 27, 223-234 (1972)

6. Achieser, N.I., Glasman,I.M.: Theorie der linearen Operatoren im Hilbert-Raum. Berlin: Akademie Verlag 1954

7. Maurin, K.: General eigenfunction expansions and unitary representations of topological groups. Warszawa: Polish Scientific Publishers 1968 
8. Maurin, K.: Bull. Acad. Pol. Sci., Ser. sci. math. 11, 115-123 (1963)

9. Bratteli, O.: Commun. Pure Appl. Math. XXV, 759-779 (1972)

10. Powers, R.: Commun. math. Phys. 21, 85-124 (1971)

11. Kelley, J.L.: General topology. Princeton: Van Nostrand 1955

12. Gel'fand, I. M., Vilenkin, N. Ya.: Generalized functions, Vol. 4, New York: Academic Press 1964

13. Dixmier, J.: Les algebres d'opérateurs dans l'espace hilbertien. Paris: Gautier-Villars 1957

14. Lassner, G.: Rep. Math. Phys. 3, 279-293 (1972)

15. Pietsch,A.: Nuclear locally convex spaces. Berlin-Heidelberg-New York: Springer 1972

16. Ruelle, D.: Helv. Phys. Acta 35, 147 (1962)

17. Araki, H.: Progr. Theor. Phys. 32, 844-854 (1964)

18. Borchers, H. J.: Commun. math. Phys. 1, 49-56 (1965)

Communicated by K. Hepp and H. Araki

\author{
H. J. Borchers \\ J. Yngvason \\ Institut für Theoretische Physik \\ Universität Göttingen \\ D-3400 Göttingen \\ Bunsenstraße 9 \\ Federal Republic of Germany
}

